\title{
Hyperbolic cusps with convex polyhedral boundary
}

\author{
FRANÇOIS FILLASTRE \\ IVAN IZMESTIEV
}

\begin{abstract}
We prove that a 3-dimensional hyperbolic cusp with convex polyhedral boundary is uniquely determined by the metric induced on its boundary. Furthermore, any hyperbolic metric on the torus with cone singularities of positive curvature can be realized as the induced metric on the boundary of a convex polyhedral cusp.

The proof uses the discrete total curvature functional on the space of "cusps with particles", which are hyperbolic cone-manifolds with the singular locus a union of half-lines. We prove, in addition, that convex polyhedral cusps with particles are rigid with respect to the induced metric on the boundary and the curvatures of the singular locus.
\end{abstract}

Our main theorem is equivalent to a part of a general statement about isometric immersions of compact surfaces.

57M50; 53C24

\section{Introduction}

In Section 1.1 we state the results, Section 1.2 puts them in a more general context, and Section 1.3 gives a sketch of the proof and a plan of the paper. Precise definitions will be given in Section 2.

\subsection{Statements}

Let $M \approx \mathbb{T} \times[0,+\infty)$ be a convex hyperbolic 3 -manifold with a cusp and with piecewise geodesic boundary. We call it a convex polyhedral cusp; see Definition 2.1 for a precise definition. The induced metric on $\partial M$ is a hyperbolic metric on the torus $\mathbb{T}$ with conical singularities of positive singular curvature. The main result of this paper is that the metric on $M$ is uniquely determined by the metric on $\partial M$ :

Theorem A Let $g$ be a hyperbolic metric with conical singularities of positive singular curvature on the 2-torus $\mathbb{T}$. Then there exists a convex polyhedral cusp $M$ such that $\partial M$ with the induced metric is isometric to $(\mathbb{T}, g)$. Furthermore, $M$ is unique up to isometry. 
This theorem can be viewed as a statement about isometric immersions. A convex parabolic polyhedron is a pair $(P, G)$, where $P$ is a convex polyhedron in $\mathbb{H}^{3}$, and $G$ is a discrete subgroup of $\operatorname{Iso}^{+}\left(\mathbb{H}^{3}\right)$ that acts freely cocompactly on a horosphere and leaves $P$ invariant. Figure 1 shows an example of a convex parabolic polyhedron, whose vertices form an orbit of the group $G$. For any convex parabolic polyhedron $(P, G)$, the quotient $P / G$ is a convex polyhedral cusp. Conversely, the universal cover of a convex polyhedral cusp is isometric to a convex parabolic polyhedron. Thus Theorem A says that each hyperbolic metric on $\mathbb{T}$ with conical singularities of positive singular curvature can be uniquely realized as the boundary of a convex parabolic polyhedron:

Theorem A' Let $g$ be a hyperbolic metric with conical singularities of positive singular curvature on the torus $\mathbb{T}$. Then there exists a unique up to ambient isometry convex parabolic polyhedron $(P, G)$ such that $\partial P / G$ is isometric to $(\mathbb{T}, g)$.

This is a part of a general statement about polyhedral realization of metrics on compact surfaces; see Section 1.2.

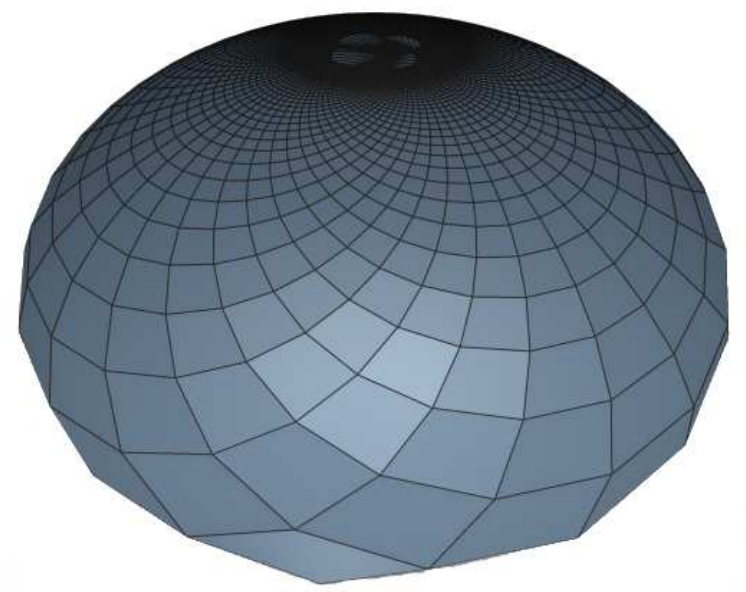

Figure 1: A simplest convex parabolic polyhedron in the Klein projective model

The uniqueness part of Theorem A is a rigidity statement: two convex polyhedral cusps with isometric boundaries are isometric. Compare this with the Cauchy-Alexandrov theorem on rigidity of convex polytopes $[9 ; 2]$. We prove also the corresponding infinitesimal rigidity result: any nontrivial first-order deformation of the metric on $M$ in the class of complete hyperbolic metrics induces a nontrivial first-order deformation of the metric on $\partial M$. 


\section{Theorem B Convex polyhedral cusps are infinitesimally rigid.}

\section{Theorem B' Convex parabolic polyhedra are parabolically infinitesimally rigid.}

For definition of parabolic infinitesimal rigidity see Section 5.3.

Our method involves study of convex polyhedral cusps with particles. These are conemanifolds that are very much like convex polyhedral cusps but have cone singularities along half-lines (the particles) that start at the cone singularities of the boundary.

For cusps with particles we prove a global rigidity statement:

Theorem C Two convex polyhedral cusps with particles with the same metric on the boundary and the same singular curvatures are isometric.

\subsection{Related work}

1.2.1 Towards a general realization statement Theorem A' is similar to a famous theorem of A D Alexandrov:

Theorem 1.1 (A D Alexandrov [1;2]) Let $g$ be a metric of constant curvature $K$ with conical singularities of positive singular curvature on the 2 -sphere $\mathbb{S}$. Then $(\mathbb{S}, g$ ) can be realized as a convex polyhedral surface in the 3-dimensional Riemannian space-form of curvature $K$. The realization is unique up to an ambient isometry.

Clearly, the positivity condition on the singular curvatures is necessary if one wants to realize the given metric on a convex polyhedral surface in a Riemannian space-form. In Lorentzian space-forms, convex space-like polyhedral surfaces can have singularities of negative singular curvature.

Theorem 1.2 (Rivin [24], Rivin-Hodgson [16]) Let $g$ be a spherical metric with negative cone singularities on $\mathbb{S}$ and lengths of closed geodesics greater than $2 \pi$. Then $(\mathbb{S}, g)$ can be uniquely realized as a convex polyhedral surface in de Sitter space.

Actually, the uniqueness statement proved in [16] is slightly weaker; see Schlenker [29]. Given a surface of genus higher than one equipped with a cone metric, one can try to realize its universal cover as the boundary of a so called convex Fuchsian polyhedron. Corresponding realization theorems are proved by the first author [12] for hyperbolic space and by Schlenker [26] and the first author [11] for Lorentzian space-forms.

The remaining case of metrics on the torus has two parts: hyperbolic metric with positive cone singularities and spherical metric with negative cone singularities. The former is done in the present paper, whereas the latter is the subject of a forthcoming paper [14]. Putting all together leads to a solution of the following problem. 
Problem 1 Let $g$ be a metric of constant curvature $K$ with conical singularities of a constant sign $\varepsilon \in\{-,+\}$ on a compact surface $S$. In the case $K=1, \varepsilon=-$ we require the lengths of contractible geodesics to be $>2 \pi$. Can the universal cover of $(S, g)$ be uniquely realized in $M_{K}^{\varepsilon}$ as a convex polyhedral surface invariant under the action of a representation of $\pi_{1}(S)$ in a subgroup of $\mathrm{Iso}^{+}\left(M_{K}^{\varepsilon}\right)$ acting freely cocompactly on a totally umbilical surface?

Here $M_{K}^{+}$is the Riemannian space-form of curvature $K$, and $M_{K}^{-}$is the Lorentzian space-form of curvature $K$. See also the introduction in [12].

As an extension of Problem 1, one can allow the surface $S$ to have boundary, and, in the case $K=-1$ the metric $g$ to have cusps. The case of a hyperbolic metric with positive cone singularities, cusps and geodesic boundary was done by the first author [13]. The case of a Euclidean metric on a disk with positive cone singularities and convex piecewise geodesic boundary is Alexandrov's convex cap theorem; see Alexandrov [2], Volkov [34] and Izmestiev [18].

A more traditional way to state Theorem 1.2 is in terms of the dual metric of a convex hyperbolic polyhedron, which is obtained with the help of the Gauss map [16]. If the combinatorics of the polyhedron is known, then the dual metric is defined by the values of the dihedral angles. This implies Andreev's Theorem [4] about compact acute-angled hyperbolic polytopes; see Hodgson [15].

A motivation for Alexandrov's theorem was its smooth counterpart known as Weyl's problem. There, $g$ is a Riemannian metric on the 2 -sphere $\mathbb{S}$ with Gaussian curvature of $g$ greater than $K$ everywhere. The case of smooth metrics on surfaces of genus higher than one was done by Schlenker [20]. The case of smooth metrics on the torus (the smooth analog of our Theorem A and of the result in our forthcoming paper [14]) is not done, as far as we know. However, Theorems 0.1 and 0.2 from Schlenker [30] imply that a different realization is possible: metrics on the torus with appropriate bounds on the Gaussian curvature can be realized as the induced metric, respectively dual metric, of the boundary of a solid torus equipped with hyperbolic metric.

\subsubsection{Hyperbolic manifolds with convex polyhedral boundary Here we restrict} our attention to the hyperbolic cases of theorems above. A reformulation of Theorem 1.1 is that each hyperbolic cone metric on the sphere with singularities of positive curvature can be uniquely extended to a hyperbolic metric with a convex polyhedral boundary on the ball.

In the same way, the hyperbolic realization theorem for genus $>1$ by the first author [12] says that the metric inside a "Fuchsian manifold" with convex polyhedral boundary 
is uniquely determined by the metric on the boundary. Both are special cases of the following statement.

Problem 2 Let $M$ be a compact connected 3-manifold with boundary, and let $M$ admit a complete hyperbolic convex cocompact metric. Can each hyperbolic cone metric on $\partial M$ with singularities of positive curvature be uniquely extended to a hyperbolic metric on $M$ with convex polyhedral boundary?

In the case of smooth strictly convex boundary the analog was proved in Schlenker [30] (the case of the ball should follow from the works of Alexandrov and Pogorelov). In both polyhedral and smooth cases the same problem can be posed for geometrically finite manifolds. Theorem A provides the simplest polyhedral case of such generalization. Similar questions can be posed about the dual metric on the boundary. In the smooth compact case the dual metric is simply the third fundamental form, and the problem was solved also by Schlenker [28; 30] and Labourie and Schlenker [20].

1.2.3 Manifolds with particles The term "manifold with particles" comes from the physics literature, where the manifolds are Lorentzian and the singularities are along time-like geodesics. The definition can be naturally extended to certain hyperbolic conemanifolds with singularities along infinite lines; see eg Krasnov and Schlenker [19], Moroianu and Schlenker [23] and Bonsante and Schlenker [7]. By analogy, we have adopted the same terminology for our "cusps with particles".

Theorem $\mathrm{C}$ states that a convex polyhedral cusp with particles is uniquely determined by the metric on its boundary and the singular curvatures along the particles. One can ask what boundary metric and particles curvatures can be realized.

Problem 3 Let $g$ be a hyperbolic cone metric on $\mathbb{T}$ with $n$ singularities of positive curvature. What are the necessary and sufficient conditions on the numbers $\kappa_{1}, \ldots, \kappa_{n}$ so that there exists a cusp with particles of curvatures $\kappa_{1}, \ldots, \kappa_{n}$ and with convex polyhedral boundary isometric to $g$ ?

One obvious condition on $\left(\kappa_{i}\right)$ is $\sum_{i=1}^{n} \kappa_{i}=0$; see Lemma 2.11 .

\subsubsection{Weakly convex star-shaped parabolic polyhedra A star-shaped parabolic} polyhedron is a pair $(P, G)$, where $P \subset \mathbb{H}^{3}$ is the cone with the apex $c \in \partial \overline{\mathbb{H}^{3}}$ over a polyhedral surface that projects bijectively onto horospheres with center $c$, and $G$ is a discrete subgroup of Iso ${ }^{+}\left(\mathbb{H}^{3}\right)$ that acts freely cocompactly on horospheres with center $c$ and leaves $P$ invariant. Clearly, every convex parabolic polyhedron is star-shaped, but the converse does not hold. 
A star-shaped parabolic polyhedron is called weakly convex if its vertices are vertices of some convex polyhedron.

By using the argument of Schlenker [27], we prove the following theorem.

Theorem D Weakly convex star-shaped parabolic polyhedra are parabolic infinitesimally rigid.

\subsection{Sketch of the proof and plan of the paper}

We prove Theorem A by the variational method. The variational method consists in identifying the object we are looking for with a critical point of a functional. If a concave functional on a convex domain attains its maximum in the interior, then the maximum point is the unique critical point. This yields both the existence and uniqueness statement for the desired object.

The domain that we consider is the space $\mathcal{M}(\mathbb{T}, g)$ of convex polyhedral cusps with particles and with boundary $(\mathbb{T}, g)$. A cusp with particles is glued from semi-ideal pyramids with the common ideal apex so that the pyramids allow a consistent truncation by horospheres. A truncation yields a collection $\left(h_{i}\right)_{i \in \Sigma}$ of truncated particle lengths, one for each singular point $i \in \Sigma$. Change of a truncation results in adding a common constant to all of the $h_{i}$. We call the corresponding equivalence class $[h]$ the particle lengths.

Section 3 contains two important results. First, we show that for a given metric $g$ on the boundary, a convex polyhedral cusp with particles is uniquely determined by its particle lengths $[h]$. That is to say, no two convex cusps with particles with different face structures have the same particle lengths. Second, we show that $\mathcal{M}(\mathbb{T}, g)$ is a compact convex subset of $\mathbb{R}^{\Sigma} /\langle\mathbf{1}\rangle$, where $\langle\mathbf{1}\rangle$ is the vector 1 -space spanned by $(1,1, \ldots, 1) \in \mathbb{R}^{\Sigma}$.

The functional on $\mathcal{M}(\mathbb{T}, g)$ is given by the formula

$$
S(M)=-2 \operatorname{Vol}(M)+\sum h_{i} \kappa_{i}+\sum \ell_{e}\left(\pi-\theta_{e}\right) .
$$

Here the first sum ranges over all singularities of the metric $g$, and $\kappa_{i}$ denotes the singular curvature at the $i$-th particle. The sum does not depend on the choice of a truncation due to $\sum \kappa_{i}=0$. The second sum is of a similar nature: here $\ell_{e}$ is the length of a boundary edge $e$, and $\theta_{e}$ is the dihedral angle at this edge. Functional $S$ is the discrete analog of the total scalar curvature, which is also known as the Hilbert-Einstein functional. 
Schläfli's formula implies

$$
\frac{\partial S}{\partial h_{i}}=\kappa_{i}
$$

Thus a critical point of $S$ corresponds to a convex polyhedral cusp with vanishing curvatures of particles. An explicit computation of derivatives shows that the Hessian of $S$ is negatively semidefinite. Although at some points the Hessian might be degenerate, it turns out that the functional $S$ is strictly concave on $\mathcal{M}(\mathbb{T}, g)$. The functional $S$ is investigated in Section 4.

Proofs of Theorem A and Theorem D are given is Section 5. All of them use either the nondegeneracy of the Hessian or the strict concavity of $S$.

\subsection{Remarks}

In the physics literature, the functional $\sum h_{i} \kappa_{i}$ for a manifold built up from Euclidean simplices is known as the Regge functional. In mathematics, the boundary term $\sum \ell_{e}\left(\pi-\theta_{e}\right)$ appeared in the works of Steiner and Minkowski. Minkowski also showed that this is the correct discrete analog of the total mean curvature of the boundary of a convex body. In the smooth case, Blaschke and Herglotz [5] suggested to use the Hilbert-Einstein functional to approach Weyl's problem, which is a smooth analog of Alexandrov's theorem in $\mathbb{R}^{3}$ : show that any convex Riemannian metric on the sphere is uniquely realized as the boundary of a convex body. Recently, Michael Anderson [3] proposed an approach to the geometrization of 3-manifolds via scalar curvature type functionals.

The variational method used in the present paper was earlier applied by the second author [18] to prove the existence and uniqueness of a Euclidean convex cap with given metric on the boundary. Functional $S$ was also used by Bobenko and the second author [6] to give a new proof of Alexandrov's theorem in $\mathbb{R}^{3}$. In [6], the matter was complicated by the fact that $S$ was neither concave nor convex.

An alternative method of proving realization statements like Theorems 1.1 and 1.2 (see also the first author's papers [12;11]) is the deformation method, also known as Alexandrov's method. The idea is to consider the map between the space of convex polyhedral surfaces and the space of cone metrics that associates to a surface its induced metric. The key point is to prove the local rigidity: a deformation of a surface always induces a deformation of a metric. In other words, the map "induced metric" is a local homeomorphism. Then, by topological arguments, this map is shown to be a global homeomorphism. Note a different role of the infinitesimal rigidity in the two approaches. Being a key lemma in the deformation method, it is a byproduct in the variational method (nondegeneracy of the Hessian at a critical point). 
The variational method is constructive: a computer program can be written that finds the critical point of a functional numerically. For Alexandrov's theorem in $\mathbb{R}^{3}$, such a program was created by Stefan Sechelmann [31].

Acknowledgments We are grateful to Cyril Lecuire and Jean-Marc Schlenker for useful and interesting conversations and to the referee for valuable suggestions. We thank Stefan Sechelmann for making Figure 1.

Visits of the first author to the TU Berlin and of the second author to the University of Neuchâtel have facilitated the collaboration. We thank both institutions for hospitality.

The first author was partially supported by Schweizerischer Nationalfonds 200020113199/1. The second author was supported by the DFG Research Unit 565 "Polyhedral Surfaces".

\section{Definitions and preliminaries}

In Section 2.1 we define convex polyhedral cusps. These are hyperbolic cusps whose metric in the neighborhood of boundary points is modelled on convex polyhedral cones. A convex polyhedral cone is the intersection of finitely many halfspaces in $\mathbb{H}^{3}$ whose boundary planes pass through one point. Then we define convex parabolic polyhedra and show that they are universal covers of convex polyhedral cusps. In Section 2.2 we define hyperbolic cusps with particles as cone-manifolds glued from semi-ideal pyramids. Finally, Section 2.3 contains some hyperbolic geometry needed in the sequel.

\subsection{Cusps and parabolic polyhedra}

Definition 2.1 A hyperbolic cusp with boundary is a complete hyperbolic manifold of finite volume homeomorphic to $\mathbb{T} \times[0,+\infty)$. We say that the cusp has a convex polyhedral boundary if every point on the boundary has a neighborhood isometric to a neighborhood of a point on the boundary of a convex polyhedral cone in $\mathbb{H}^{3}$.

We call hyperbolic cusps with convex polyhedral boundary briefly convex polyhedral cusps. Clearly, the induced metric on the boundary of a convex polyhedral cusp is a hyperbolic metric with cone singularities of positive curvature. It is easy to define vertices, edges and faces of a convex polyhedral cusp. Vertices are exactly the cone singularities of the metric on the boundary. Every edge is a geodesic joining the vertices. Edges cut the boundary $\partial M$ of the cusp $M$ into faces, which are maximal connected open subsets of $\partial M$ that bound $M$ geodesically. 
Definition 2.2 A convex parabolic polyhedron in $\mathbb{H}^{3}$ is a pair $(P, G)$, where $P \subset \mathbb{H}^{3}$ is the convex hull of a discrete set of points, and $G$ is a discrete subgroup of $\operatorname{Iso}^{+}\left(\mathbb{H}^{3}\right)$ that acts freely cocompactly on a horosphere in $\mathbb{H}^{3}$ and leaves $P$ invariant.

Clearly, the vertex set of $P$ is $G$-invariant. Since it is discrete, it is the union of finitely many orbits of the group $G$. The simplest example of a convex parabolic polyhedron is the convex hull of one orbit; see Figure 1.

The group $G$ has a unique fixed point $c$ in $\partial \overline{\mathbb{H}^{3}}$. Clearly, $c$ lies in the closure of $P$. We call $c$ the center of the polyhedron $P$.

Lemma 2.3 Let $(P, G)$ be a convex parabolic polyhedron. Then the quotient space $P / G$ is a convex polyhedral cusp.

Proof The group $G$ acts on a horosphere by orientation preserving Euclidean isometries. Since this action is by assumption free and cocompact, we have $G \cong \mathbb{Z}^{2}$ and $B / G \approx \mathbb{T} \times[0,+\infty)$ for any horoball $B$ centered at $c$. Since the vertex set of $P$ is the union of finitely many orbits, there are horoballs $B_{1}$ and $B_{2}$ centered at $c$ such that $B_{1} \subset P \subset B_{2}$. It is easy to see that any geodesic passing through $c$ intersects the boundary of $P$ at exactly one point. It follows that $P / G$ is homeomorphic to $\mathbb{T} \times[0,+\infty)$. From $P \subset B_{2}$ it also follows that $P / G$ has finite volume. The manifold $P / G$ is complete since it is a closed subset of a complete manifold $B_{2} / G$. Finally, $P / G$ has convex polyhedral boundary because $P$ has.

Let $M$ be a convex polyhedral cusp. By definition it is locally convex, hence it is convex [8, Corollary I.1.3.7.]. It follows that the developing map $D: \widetilde{M} \rightarrow \mathbb{H}^{3}$ is an isometric embedding [8, Proposition I.1.4.2.]. The action of the fundamental group $\pi_{1} M \cong \mathbb{Z}^{2}$ on $\widetilde{M}$ by deck transformations yields a representation $\rho: \pi_{1} M \rightarrow \operatorname{Iso}^{+}\left(\mathbb{H}^{3}\right)$.

Lemma 2.4 The pair $\left(D(\widetilde{M}), \rho\left(\pi_{1} M\right)\right)$ is a convex parabolic polyhedron.

Proof Clearly, $D(\widetilde{M})$ is a convex polyhedron homeomorphic to the half-space. Its vertices form a discrete set, because they correspond to vertices of $M$, whose number is finite.

The thin part of $M$ contains a totally umbilic torus $C$ with Euclidean metric. It follows that the developing map maps the universal cover of $C$ to a horosphere. The group $\rho\left(\pi_{1} M\right)$ acts on $D(\widetilde{C})$ freely with a compact orbit space $C$. The lemma follows. $\square$

Corollary 2.5 Every face of a convex polyhedral cusp is a convex hyperbolic polygon.

Lemma 2.3 and Lemma 2.4 imply that $(P, G) \mapsto P / G$ is a one-to-one correspondence between the equivariant isometry classes of convex parabolic polyhedra and isometry classes of convex polyhedral cusps. Thus Theorem A is equivalent to Theorem A'. 


\subsection{Cusps with particles}

Definition 2.6 A semi-ideal pyramid in $\mathbb{H}^{3}$ is the convex hull of a convex polygon $A$ and a point $a \in \partial \overline{\mathbb{H}^{3}}$ such that $a$ is not coplanar to $A$. The point $a$ is called the apex of the pyramid, the polygon $A$ its base.

A convex polyhedral cusp can be decomposed into semi-ideal pyramids with a common apex. Indeed, let $M$ be a cusp and let $(P, G)$ be the corresponding parabolic polyhedron. If $c \in \partial \overline{\mathbb{H}^{3}}$ is the center of $P$, then $P$ is composed from semi-ideal pyramids with the apex $c$ over the faces of $P$. Clearly, this decomposition of $P$ descends to a decomposition of $M \cong P / G$. In the example on Figure 1, the decomposition of $M$ consists of a single isosceles quadrangular pyramid whose faces are identified according to the standard gluing of a torus from a parallelogram.

Let us see when a gluing of pyramids defines a convex polyhedral cusp.

Definition 2.7 A cuspidal complex is a collection of semi-ideal pyramids glued isometrically along some pairs of faces so that combinatorially the gluing is represented by the cone with an ideal apex over a polyhedral decomposition of the torus.

If the pyramids of a cuspidal complex fit well around their lateral edges, then the result of the gluing is a hyperbolic manifold with polyhedral boundary. This manifold can be noncomplete as the following example shows. In the Poincaré half-space model, take a semi-ideal pyramid with vertices $(1,0,1),(0,1,1),(2,0,2),(0,2,2)$, and the point at infinity as the apex. Clearly, the semi-ideal triangles in each pair of opposite sides of the pyramid are isometric. When we identify them, we get a noncomplete manifold homeomorphic to $\mathbb{T} \times[0,+\infty)$.

For a semi-ideal pyramid $\Delta$, choose a horoball $B$ centered at the apex of the pyramid and disjoint with its base. The body $\Delta \backslash B$ is called a truncated semi-ideal pyramid or a horoprism.

Definition 2.8 A cuspidal complex is called compatible if every pyramid of the complex can be truncated so that the gluing isometries restrict to the faces of the truncated pyramids.

Lemma 2.9 The manifold defined by a cuspidal complex is complete if and only if the complex is compatible. 
Proof If the manifold is complete, then its thin part contains a cusp with totally umbilic Euclidean boundary. Cutting this cusp off defines a compatible truncation of the pyramids.

Conversely, assume that the complex is compatible. For every pyramid $\Delta_{i}$ consider the corresponding horoball sectors $\Delta_{i} \cap B_{i}$. It is easy to see that the developing map maps the union $\bigcup_{i}\left(\Delta_{i} \cap B_{i}\right)$ to a horoball in $\mathbb{H}^{3}$. Thus the manifold $\bigcup_{i}\left(\Delta_{i} \cap B_{i}\right)$ is complete and so is the whole manifold defined by the complex.

In general, a compatible cuspidal complex defines a cone-manifold whose singular locus is contained in the union of half-lines that come from the lateral edges of the semi-ideal pyramids. We call these half-lines particles.

Definition 2.10 A polyhedral cusp with particles is a hyperbolic cone-manifold defined by a compatible cuspidal complex. A polyhedral cusp with particles is called convex if and only if the total dihedral angle at every boundary edge is at most $\pi$. For a boundary singularity $i$, denote by $\omega_{i}$ the total dihedral angle around the $i$-th particle. The curvature of the $i$-th particle is defined as

$$
\kappa_{i}=2 \pi-\omega_{i} .
$$

A truncated polyhedral cusp with particles is defined in the same way as a polyhedral cusp with particles, using horoprisms instead of semi-ideal pyramids.

Lemma 2.11 The curvatures of a convex polyhedral cusp with particles satisfy

$$
\sum_{i \in \Sigma} \kappa_{i}=0
$$

Proof Truncate the cusp. The induced metric on the surface of truncation is a flat metric with conical singularities on the torus. Clearly, the curvatures of the singularities are exactly the $\kappa_{i}$. The lemma follows from the Gauss-Bonnet formula.

We are interested in the cusps whose boundary is isometric to $(\mathbb{T}, g)$, where $g$ is a hyperbolic metric with conical singularities of positive singular curvatures on the torus $\mathbb{T}$.

Definition 2.12 We denote by $\mathcal{M}(\mathbb{T}, g)$ the set of convex polyhedral cusps with particles $M$ with $\partial M=(\mathbb{T}, g)$. By $\mathcal{M}_{\mathrm{tr}}(\mathbb{T}, g)$ we denote the set of truncated convex polyhedral cusps with particles with the boundary $(\mathbb{T}, g)$. 
Formally speaking, an element of $\mathcal{M}(\mathbb{T}, g)$ is a pair $(M, f)$, where $f: \partial M \rightarrow(\mathbb{T}, g)$ is an isometry. It will be convenient to identify $\partial M$ with the given metric torus $(\mathbb{T}, g)$, so that we can omit mentioning $f$.

Theorem $\mathrm{A}$ is equivalent to say that in $\mathcal{M}(\mathbb{T}, g)$ there is a unique cusp with vanishing curvatures of particles. Note that we don't fix an isometry between $\partial M$ and $(\mathbb{T}, g)$ in Theorem A. In this case, it does not really matter because the uniqueness is stated.

As in the case of a convex polyhedral cusp, the boundary of a convex polyhedral cusp with particles consists of vertices, edges and faces. Unlike the case without particles, faces of a cusp with particles can be non-simply connected, and there can be isolated vertices, as the following example shows.

Example In the Poincaré half-space model, take the point $a=(0,0,1)$ and points $b, c$ on the Euclidean unit sphere centered at 0 so that $b$ and $c$ lie at an equal distance from $a$ and the angle at the vertex $a$ in the spherical triangle $a b c$ is $<\frac{\pi}{2}$. The semi-ideal pyramid with the base $a b c$ and the apex at the point at infinity has dihedral angles $\frac{\pi}{2}$ at the edges $a b$ and $a c$ and an angle $<\frac{\pi}{2}$ at $b c$. Take four copies of this pyramid and glue them cyclically around the edge $a \infty$. The result is a semi-ideal quadrangular pyramid with a particle. By identifying the pairs of its opposite sides, we obtain a convex polyhedral cusp with particles. Its boundary contains two vertices, two loop edges and a single face that looks as a punctured square.

More technically, an element of $\mathcal{M}_{\mathrm{tr}}(\mathbb{T}, g)$ is defined by a subdivision of $(\mathbb{T}, g)$ and by lengths of lateral edges of horoprisms over the faces of the subdivision. This is spelled out in the following definition.

Definition 2.13 Let $M_{\mathrm{tr}} \in \mathcal{M}_{\mathrm{tr}}(\mathbb{T}, g)$ be a truncated convex polyhedral cusp with particles. Let $T$ be a triangulation of $(\mathbb{T}, g)$ that refines the natural decomposition of the boundary $\partial M_{\mathrm{tr}}$, and let $h_{i}$ be the length of the truncated particle with the endpoint $i \in \Sigma$, where $\Sigma$ is the set of singularities of $g$. We associate to $M_{\text {tr }}$ the pair $(T, h)$, where $h$ stands for $\left(h_{i}\right)_{i \in \Sigma}$.

Similarly, to every $M \in \mathcal{M}(\mathbb{T}, g)$ we associate a pair $(T,[h])$, where $(T, h)$ represents a truncation of $M$, and $[h]$ is the equivalence class under the relation $h \sim h^{\prime} \Leftrightarrow h_{i}^{\prime}=h_{i}+c$ for all $i$ and some constant $c$.

The equivalent class $[h]$ is called the particle lengths of $M$. The cusp $M$ is isosceles if it is made of isosceles semi-ideal pyramids, ie if $[h]=[0, \ldots, 0]$.

By a triangulation we mean a decomposition of $(\mathbb{T}, g)$ into open hyperbolic triangles by geodesic arcs (edges of the triangulation) with endpoints in $\Sigma$. We don't impose 
any restrictions on the combinatorics, so that there may be loops and multiple edges, and two triangles may have two edges in common, and two edges of a triangle may be identified. An edge with endpoints $i$ and $j$ is denoted by $i j$, a triangle with vertices $i, j$ and $k$ is denoted by $i j k$. Because of what we just said, different edges or triangles may obtain the same notation, and some letters in the notation may repeat. But this should not lead to confusion.

\subsection{Some hyperbolic trigonometry}

Lemma 2.14 (Cosine law for semi-ideal triangles) Let $B$ be a horodisk in the hyperbolic plane and let $i, j$ be two points not in $B$. Let $h_{i}, h_{j}, \lambda$ be the distances $\operatorname{dist}(i, B), \operatorname{dist}(j, B), \operatorname{dist}(i, j)$, respectively, and let $\rho_{i}$ be the angle between the geodesic segment $i j$ and the perpendicular from $i$ to $B$. Then

$$
\cos \rho_{i}=\frac{\cosh \lambda-e^{h_{j}-h_{i}}}{\sinh \lambda} .
$$

Proof Go to the limit in the cosine law for the triangle with vertices $i, j$ and third vertex approaching the center of the horodisk.

Lemma 2.15 Let $B$ be a horodisk in the hyperbolic plane, and $L$ be a line disjoint with $B$. Then for every $x \in L$ we have

$$
\operatorname{dist}(x, B)=\log \cosh (\operatorname{dist}(x, a))+\operatorname{dist}(a, B),
$$

where $a$ is the point on $L$ nearest to $B$.

Proof Consider the semi-ideal triangle with vertices $a, x$ and the center $c(B)$ of $B$. Apply Equation (1), taking $a$ for $i, x$ for $j$. We have $\cos \rho_{i}=0$ and $\lambda=\operatorname{dist}(x, a)$. Hence

$$
\cosh (\operatorname{dist}(x, a))=e^{h_{j}-h_{i}}=\exp (\operatorname{dist}(x, B)-\operatorname{dist}(a, B)),
$$

and the claim follows.

Lemma 2.16 Let $i, j, k$ be three collinear points in $\mathbb{H}^{2}$ such that $j$ lies between $i$ and $k$. For a horodisk $B$ that contains none of the points $i, j, k$, denote by $h_{i}, h_{j}, h_{k}$ the distances $\operatorname{dist}(i, B), \operatorname{dist}(j, B), \operatorname{dist}(k, B)$, respectively. Then

$$
e^{h_{j}}=\frac{\sinh \mu}{\sinh (\lambda+\mu)} e^{h_{i}}+\frac{\sinh \lambda}{\sinh (\lambda+\mu)} e^{h_{k}},
$$

where $\lambda=\operatorname{dist}(i, j), \mu=\operatorname{dist}(j, k)$.

Proof Let $c$ be the center of $B$, and let $\rho_{j}, \pi-\rho_{j}$ be the angles between the geodesic $i k$ and the perpendicular from $j$ to $\partial B$. Compute $\cos \rho_{j}$ by the Equation (1) from the semi-ideal triangles $i j c$ and $j k c$ and equate the two expressions. 


\section{The space of convex polyhedral cusps with particles}

In Section 3.1 we show that a convex polyhedral cusp with particles is uniquely determined by the particle lengths $[h]$ introduced in Definition 2.13 . This identifies the space of cusps $\mathcal{M}(\mathbb{T}, g)$ with a subset of $\mathbb{R}^{n-1}$, where $n$ is the number of singularities of $g$. Section 3.2 contains several lemmas that are later used in Section 3.3 to prove Proposition 3.15. The proposition says that $\mathcal{M}(\mathbb{T}, g)$ is a compact convex subset of $\mathbb{R}^{n-1}$.

Everywhere in this section we mean by a cusp a cusp with particles with polyhedral boundary.

\subsection{Particle lengths define a cusp}

Recall that a truncated convex cusp was defined as a union of horoprisms. A horoprism is a semi-ideal pyramid with a neighborhood of the ideal vertex cut off along a horosphere. A horoprism has a hyperbolic base and a Euclidean base. The lateral edges of a horoprism are orthogonal to its Euclidean base. The lengths of lateral edges are called heights of the horoprism. Clearly, a horoprism is uniquely determined by the hyperbolic base and the heights. In what follows, we consider only triangular horoprisms.

Cutting a truncated convex cusp into triangular horoprisms produces a pair $(T, h)$, where $T$ is a geodesic triangulation of $(\mathbb{T}, g)$, and $h=\left(h_{i}\right)_{i \in \Sigma}$ is the collection of heights of the horoprisms, which at the same time are the truncated particle lengths in the cusp. Occasionally, there is some freedom in the choice of $T$, since it may be any refinement of the canonical face decomposition of the cusp boundary.

Our goal is to prove:

Proposition 3.1 A truncated convex cusp is uniquely determined by its truncated particle lengths.

In other words, if $(T, h)$ and $\left(T^{\prime}, h\right)$ are pairs associated with the truncated convex cusps $M_{\mathrm{tr}}, M_{\mathrm{tr}}^{\prime}$, respectively, then $M_{\mathrm{tr}}=M_{\mathrm{tr}}^{\prime}$.

The following definition introduces a concept that will be used through the whole section.

Definition 3.2 The distance function of a truncated convex cusp $M_{\mathrm{tr}}$ is a map $(\mathbb{T}, g) \rightarrow$ $\mathbb{R}$ that associates to every point on the hyperbolic boundary of $M_{\text {tr }}$ its distance from the Euclidean boundary. 
Due to Lemma 2.15 we know that in the interior of every face $F$ of $M_{\text {tr }}$ the distance function has the form

$$
x \mapsto \log \cosh (\operatorname{dist}(x, a))+b,
$$

where $b>0$ and $a$ is a point in $F$ or in the hyperbolic plane spanned by $F$.

We call a function of the form (2) on a subset of the hyperbolic plane a distance-like function.

Definition 3.3 A function $f:(\mathbb{T}, g) \rightarrow \mathbb{R}$ is called piecewise distance-like function, briefly $P D$ function, if there exists a geodesic triangulation $T$ of $(\mathbb{T}, g)$ such that $f$ is distance-like on every triangle of $T$.

A PD function $f$ is called $Q$-concave if for every geodesic arc $\gamma$ on $(\mathbb{T}, g)$ at every kink point of the restriction $\left.f\right|_{\gamma}$ the left derivative is greater than the right derivative.

Figure 2 shows an example of a Q-concave PD function on the line.

Recall that a triangle $i j k$ of $T$ may have identifications on the boundary; so we mean by a distance-like function on $i j k$ a function induced from a distance-like function on its development.

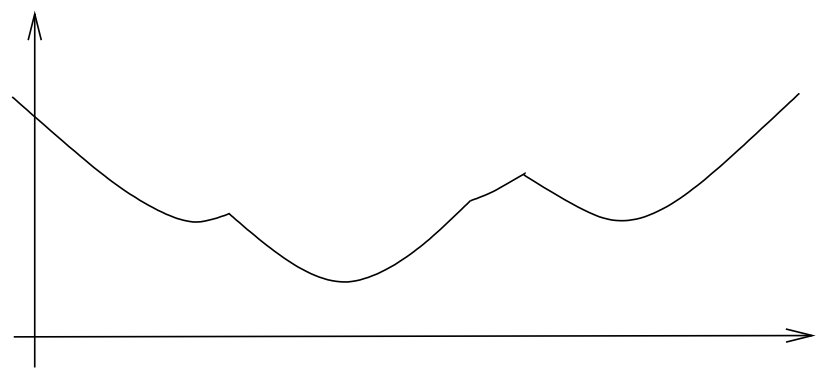

Figure 2: The graph of a Q-concave PD function

The following lemma is straightforward.

Lemma 3.4 The distance function of a truncated convex cusp is a $Q$-concave $P D$ function. Conversely, every positive $Q-$ concave $P D$ function is the distance function of a unique truncated convex cusp.

Thus we can identify the space $\mathcal{M}_{\text {tr }}(\mathbb{T}, g)$ with the space of positive Q-concave PD functions on $(\mathbb{T}, g)$. 
Definition 3.5 Let $T$ be a geodesic triangulation of $(\mathbb{T}, g)$, and let $h: \Sigma \rightarrow \mathbb{R}$ be a function on the singular set of $(\mathbb{T}, g)$. By $\widetilde{h_{T}}:(\mathbb{T}, g) \rightarrow \mathbb{R}$ we denote the PD extension of the function $h$ with respect to the triangulation $T$.

The function $\widetilde{h_{T}}$ does not always exist, but it is easy to see that if it does, then it is well-defined and unique.

Extending a function $h: \Sigma \rightarrow \mathbb{R}$ to a positive Q-concave PD function is equivalent to constructing a truncated convex cusp with truncated particle lengths $h$. Therefore Proposition 3.1 will follow from:

Lemma 3.6 Let $h$ be a function on $\Sigma$ and let $T, T^{\prime}$ be two geodesic triangulations of $(\mathbb{T}, g)$. If both functions $\widetilde{h_{T}}$ and $\widetilde{h_{T^{\prime}}}$ exist and are $Q$-concave, then they are equal.

Proof Let $x$ be an intersection point of an edge $e$ of $T$ and an edge $e^{\prime}$ of $T^{\prime}$. Then the function $\widetilde{h_{T}}$ is distance-like on $e$, and the function $\widetilde{h_{T^{\prime}}}$ is PD Q-concave on $e$. By Lemma 3.10, this implies $\widetilde{h_{T^{\prime}}}(x) \geq \widetilde{h_{T}}(x)$. Considering the edge $e^{\prime}$ instead of $e$, we get an inverse inequality. Hence $\widetilde{h_{T}}(x)=\widetilde{h_{T^{\prime}}}(x)$. The union of edges of $T$ and $T^{\prime}$ subdivides $(\mathbb{T}, g)$ into hyperbolic polygons such that both functions $\widetilde{h_{T}}$ and $\widetilde{h_{T^{\prime}}}$ are distance-like on every polygon of the subdivision. As we just proved, $\widetilde{h_{T}}(x)$ and $\widetilde{h_{T^{\prime}}}(x)$ take equal values at the vertices of the polygons. It follows that $\widetilde{h_{T}}=\widetilde{h_{T^{\prime}}}$.

Proposition 3.1 is proved. It implies that the map

$$
\begin{aligned}
\mathcal{M}_{\text {tr }}(\mathbb{T}, g) & \rightarrow \mathbb{R}^{\Sigma} \\
(T, h) & \mapsto h
\end{aligned}
$$

is an embedding. Changing a truncation of a convex cusp results in adding a common constant to all of the truncated particle lengths. Thus we have an embedding

$$
\begin{aligned}
\mathcal{M}(\mathbb{T}, g) & \rightarrow \mathbb{R}^{\Sigma} /\langle\mathbf{1}\rangle \\
(T,[h]) & \mapsto[h],
\end{aligned}
$$

where $[h]$ is an equivalence class under $\left(h_{1}, \ldots, h_{n}\right) \sim\left(h_{1}+c, \ldots, h_{n}+c\right)$.

For a geodesic triangulation $T$ of $(\mathbb{T}, g)$, denote by $\mathcal{M}_{\mathrm{tr}}^{T}(\mathbb{T}, g)$ the space of truncated convex cusps that have a representative of the form $(T, h)$. In other words, $M_{\mathrm{tr}} \in$ $\mathcal{M}_{\text {tr }}^{T}(\mathbb{T}, g)$ if and only if $M_{\text {tr }}$ can be cut into horoprisms over the triangulation $T$. We have a decomposition

$$
\mathcal{M}_{\mathrm{tr}}(\mathbb{T}, g)=\bigcup_{T} \mathcal{M}_{\mathrm{tr}}^{T}(\mathbb{T}, g) .
$$

Clearly, we have a similar decomposition for $\mathcal{M}(\mathbb{T}, g)$, where

$$
\mathcal{M}^{T}(\mathbb{T}, g)=\mathcal{M}_{\mathrm{tr}}^{T}(\mathbb{T}, g) /\langle\mathbf{1}\rangle .
$$


Let us denote by $\tilde{h}$ the distance function of the truncated convex cusp with truncated particle lengths $h=\left(h_{i}\right)_{i \in \Sigma}$. In other words, $\tilde{h}$ is the unique Q-concave PD extension of the function $h: i \mapsto h_{i}$.

\subsection{Lemmas}

We put here lemmas used in the proof of Section 3.3.

Lemma 3.7 The distance function $\tilde{h}$ of a truncated convex cusp satisfies the inequality

$$
|\tilde{h}(x)-\tilde{h}(y)|<\operatorname{dist}(x, y)
$$

for any $x, y \in \mathbb{T}$, where dist denotes the shortest path distance for the metric $g$. In particular,

$$
\max _{\mathbb{T}} \tilde{h}-\min _{\mathbb{T}} \tilde{h}<\operatorname{diam}(T, g) .
$$

Proof This follows from the fact that the gradient of the distance function is always smaller than 1 .

Lemma 3.8 The space $\mathcal{M}_{\mathrm{tr}}^{T}(\mathbb{T}, g)$ is nonempty only for finitely many geodesic triangulations $T$ of $(\mathbb{T}, g)$.

Proof The proof proceeds in two steps. First, we show that there is a constant $L$ depending on the metric $g$, such that no triangulation associated with a cusp with boundary $(\mathbb{T}, g)$ has an edge of length greater than $L$. Second, we note that there are only finitely many geodesic arcs of length $\leq L$ between points of $\Sigma$. Then the number of geodesic triangulations with edges of length $\leq L$ is also finite and we are done.

Let $\tilde{h}$ be the distance function of a truncated convex cusp, and let $e$ be an edge of an associated triangulation. The restriction of $\tilde{h}$ to $e$ is a function of the form $\log \cosh (x-a)+b$, where $x$ is the arc length parameter on $e$. It can easily be shown that for any $C \in \mathbb{R}$ there exists an $L \in \mathbb{R}$ such that

$$
\max _{e} \tilde{h}-\min _{e} \tilde{h}>C
$$

as soon as the length of $e$ is greater than $L$. Put $C=\operatorname{diam}(\mathbb{T}, g)$. Then the length of $e$ cannot be greater than $L$ due to Lemma 3.7.

The lengths of geodesic arcs between the points of $\Sigma$ form a discrete subset of $\mathbb{R}$ by the argument from [17, Proposition 1].

Remark Lemma 3.7 and Lemma 3.8 hold also for nonconvex polyhedral cusps with particles. 
Definition 3.9 For two points $i, j$ on the real line and two real numbers $h_{i}, h_{j}$, let

$$
\widetilde{h_{i j}}: \mathbb{R} \rightarrow \mathbb{R}
$$

denote the distance-like function that takes values $h_{i}, h_{j}$ at $i$ and $j$, respectively.

Note that the function $\widetilde{h_{i j}}$ exists if and only if $\left|h_{i}-h_{j}\right|<\operatorname{dist}(i, j)$.

Lemma 3.10 For any $Q$-concave $P D$ function $\tilde{h}$ on $\mathbb{R}$ such that $\tilde{h}(i)=h_{i}, \tilde{h}(j)=h_{j}$ the following holds:

$$
\begin{aligned}
& \tilde{h}(x) \geq \widetilde{h_{i j}}(x) \text { for all } x \in[i, j], \\
& \widetilde{h}(x) \leq \widetilde{h_{i j}}(x) \text { for all } x \notin[i, j] .
\end{aligned}
$$

Proof Consider the function $\tilde{h}-\widetilde{h_{i j}}$. There are numbers $x_{1}<x_{2}<\cdots<x_{n}$ and $a_{0}, b_{0}, \ldots, a_{n}, b_{n}$ such that

$$
\left.\widetilde{h}\right|_{\left[x_{m}, x_{m+1}\right]}=\log \cosh \left(x-a_{m}\right)+b_{m}
$$

for all $m$ from 0 to $n$, where we put $x_{0}=-\infty, x_{n+1}=+\infty$. Since $\log \cosh x$ is a convex function, $\left.\left(\tilde{h}-\widetilde{h_{i j}}\right)\right|_{\left[x_{m}, x_{m+1}\right]}$ is a monotone function for every $m$. Besides, since $\widetilde{h}$ is Q-concave, $\widetilde{h}-\widetilde{h_{i j}}$ is Q-concave too. Thus, if $\widetilde{h}-\widetilde{h_{i j}}$ is monotone decreasing (or constant) on $\left[x_{m-1}, x_{m}\right]$, then it is also monotone decreasing (or constant) on $\left[x_{m}, x_{m+1}\right]$. Together with

$$
\left(\widetilde{h}-\widetilde{h_{i j}}\right)(i)=\left(\widetilde{h}-\widetilde{h_{i j}}\right)(j)=0
$$

this implies that the function $\widetilde{h}-\widetilde{h_{i j}}$ is nonnegative on the interval $[i, j]$ and nonpositive outside of it. The lemma follows.

Definition 3.11 Let $\tilde{h}(x)=\log \cosh (\operatorname{dist}(x, a))+b$ be a distance-like function on a subset of $\mathbb{H}^{2}$. We call the distance $\operatorname{dist}(x, a)$ the slope of $\tilde{h}$ at $x$ and denote it by slope $_{x}(\tilde{h})$.

For a PD function $\tilde{h}$ on the torus, slope $_{x}(\tilde{h})$ is defined in an obvious way, provided that $\tilde{h}$ is locally distance-like at $x$. Clearly, slope $_{x}(\tilde{h})$ depends only on the gradient norm of $\tilde{h}$ at $x$, and it tends to $\infty$ as the gradient norm tends to 1 .

Lemma 3.12 The slopes of the convex cusps with the boundary $(\mathbb{T}, g)$ are uniformly bounded. That is, there exists a constant $D \in \mathbb{R}$ such that

$$
\operatorname{slope}_{x}(\tilde{h}) \leq D
$$

for every $Q-$ concave $P D$ function $\tilde{h}$ on $(\mathbb{T}, g)$ at every point $x \in \mathbb{T}$. 
Proof Let us show that the lemma holds for $\log \cosh D=\operatorname{diam}(\mathbb{T}, g)$. Assume the converse, and let $\tilde{h}$ and $x$ be such that slope $x(\tilde{h})>D$. On the geodesic that starts at $x$ and runs in the direction of $-\operatorname{grad}_{x}(\tilde{h})$, take the point $y$ at a distance $D$ from $x$. If we end up at a singular point before running the distance $D$, then perturb the point $x$ so that slope ${ }_{x}(\tilde{h})$ is still greater than $D$. On the geodesic arc $x y$, consider functions $\tilde{h}$ and $\widetilde{h_{x+}}$, where $\widetilde{h_{x+}}$ is the distance-like function that coincides with $\widetilde{h}$ in a neighborhood of $x$. By Lemma 3.10, we have

$$
\tilde{h}(y) \leq \widetilde{h_{x+}}(y)
$$

Due to $\operatorname{slope}_{x}(\tilde{h})>|x y|$, the function $\widetilde{h_{x+}}$ is monotone decreasing on $x y$. The convexity of $\log \cosh x$ implies

$$
\tilde{h}(x)-\widetilde{h_{x+}}(y) \geq \log \cosh |x y|=\log \cosh D=\operatorname{diam}(\mathbb{T}, g) .
$$

Therefore $\tilde{h}(x)-\tilde{h}(y) \geq \operatorname{diam}(\mathbb{T}, g) \geq \operatorname{dist}(x, y)$ which contradicts Lemma 3.7. $\square$

Let us generalize Definition 3.9 to the situation when $i, j \in \Sigma$ are singular points of $(\mathbb{T}, g)$ joined by a geodesic arc $\gamma$. Then $\widetilde{h_{i j}}$ is the distance-like function on $\gamma$ that takes values $h_{i}$ and $h_{j}$ at $i$ and $j$, respectively. Note that there are many arcs that join $i$ and $j$, so we need to specify $\gamma$ when we talk about $\widetilde{h_{i j}}$. If $h \in \mathcal{M}_{\text {tr }}(\mathbb{T}, g)$ and $\gamma$ is an edge of a triangulation associated with $h$, then $\widetilde{h_{i j}}=\left.\widetilde{h}\right|_{\gamma}$. Also we might want to extend function $\widehat{h_{i j}}$ beyond the point $i$. For this we consider a geodesic extension of $\gamma$ beyond $i$. This is a geodesic ray from $i$ that forms the angle $\pi$ with $i j$. We measure the angles around $i$ modulo the cone angle $\alpha_{i}$ at $i$, so the geodesic extension is defined for $\alpha_{i}<\pi$ as well. In general, there are two geodesic extensions ("to the left" and "to the right"), and they coincide only if $\alpha_{i}=\frac{2 \pi}{n}$ for some $n$.

Lemma 3.13 Let $i, j, k, l \in \Sigma$. Choose geodesic arcs $i k$ and $j l$ and a geodesic extension of $i k$ beyond $i$. Suppose that the extension of $i k$ intersects the arc $j l$ at a point $m$; see Figure 3. Then for every $h \in \mathcal{M}_{\mathrm{tr}}(\mathbb{T}, g)$ the following inequality holds:

$$
\widetilde{h_{i k}}(m) \geq \widetilde{h_{j l}}(m) \text {. }
$$

In particular, let $i, j \in \Sigma$ be such that there is a closed geodesic arc based at $j$ that bounds a disk in $\mathbb{T}$ such that $i$ is the only singularity inside this disk. Then for every $h \in \mathcal{M}_{\mathrm{tr}}(\mathbb{T}, g)$ the following holds:

$$
h_{i} \geq h_{j}-\log \cosh \ell_{i j}
$$

where $\ell_{i j}$ is the length of the geodesic arc $i j$ that lies inside the disk. See Figure 3. 

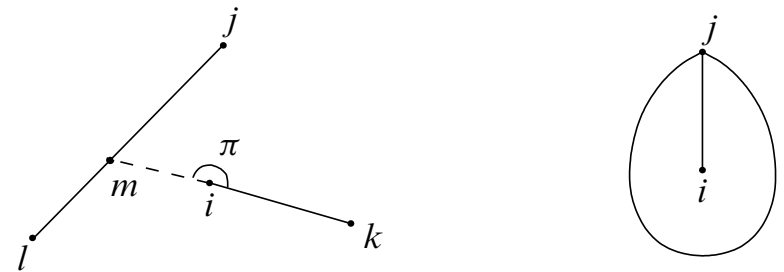

Figure 3: To Lemma 3.13

Proof Consider the restriction of the Q-concave PD function $\tilde{h}$ to the piecewise geodesic arc kim. This is a PD function, and since the arc kim can be approximated by geodesic arcs, it is also Q-concave. Then by Lemma 3.10 we have

$$
\tilde{h}(m) \leq \widetilde{h_{i k}}(m) \text {. }
$$

By Lemma 3.10 applied to the arc $j l$, we have

$$
\widetilde{h}(m) \geq \widetilde{h_{j l}}(m) .
$$

Inequality (3) follows.

Let us derive (4) from (3). Consider the function

$$
f(x)=\log \cosh (\operatorname{dist}(x, i))+h_{i}
$$

on the singular disk bounded by the arc $j j$. We have $h_{i}=f(i)$. If also $h_{j}=f(j)$, then we have

$$
\widetilde{h_{i j}}(m)=f(m)=\widetilde{h_{j j}}(m),
$$

where $m$ is the first intersection point of the geodesic extension of $i j$ beyond $i$ with the arc $j j$. It is easy to see that $\widetilde{h_{i j}}(m)$ is a monotone decreasing, and $\widetilde{h_{j j}}(m)$ is a monotone increasing function of $h_{j}$. Thus, since we have $\widetilde{h_{i j}}(m) \geq \widetilde{h_{j j}}(m)$ due to (3), we must have $h_{j} \leq f(j)$, and this is exactly the inequality (4).

Lemma 3.14 Let $i, j, k$ be three points on the real line such that $j$ lies between $i$ and $k$. Then the function $\widetilde{h_{i j}}(k)$ is a concave function of $h_{i}, h_{j}$, and the function $\widetilde{h_{i k}}(j)$ is a convex function of $h_{i}, h_{k}$.

Proof By Lemma 2.16,

$$
\widetilde{h_{i k}}(j)=\log \left(a e^{h_{i}}+b e^{h_{k}}\right)
$$

with positive $a$ and $b$. The Hessian can easily be computed and shown to be positive semidefinite. For $\widetilde{h_{i j}}(k)$ one has a similar expression with one positive and one negative coefficient. 


\subsection{Description of $\mathcal{M}(\mathbb{T}, g)$}

Here we prove the main result of this section. Recall that the space $\mathcal{M}_{\text {tr }}(\mathbb{T}, g)$ is identified with a subset of $\mathbb{R}^{\Sigma}$ by associating to a truncated cusp the truncated particle lengths $h=\left(h_{i}\right)_{i \in \Sigma}$. The space $\mathcal{M}(\mathbb{T}, g)$ is thus identified with a subset of $\mathbb{R}^{\Sigma} /\langle\mathbf{1}\rangle$.

Proposition 3.15 The space $\mathcal{M}(\mathbb{T}, g)$ of convex polyhedral cusps with particles with boundary $(\mathbb{T}, g)$ has the following properties:

(1) It is a compact convex subset of $\mathbb{R}^{\Sigma} /\langle\mathbf{1}\rangle$ with nonempty interior.

(2) If all faces of a convex cusp $M \in \mathcal{M}(\mathbb{T}, g)$ are strictly convex hyperbolic polygons (after developing on $\mathbb{H}^{2}$ ), then $M$ is an interior point of $\mathcal{M}(\mathbb{T}, g)$.

(3) For a geodesic triangulation $T$ of $(\mathbb{T}, g)$, let $\mathcal{M}^{T}(\mathbb{T}, g) \subset \mathcal{M}(\mathbb{T}, g)$ be the space of cusps whose faces are unions of triangles from $T$. Then the decomposition

$$
\mathcal{M}(\mathbb{T}, g)=\bigcup_{T} \mathcal{M}^{T}(\mathbb{T}, g)
$$

is finite, and every $\mathcal{M}^{T}(\mathbb{T}, g)$ is a compact set with piecewise analytic boundary.

Proof $\mathcal{M}(\mathbb{T}, g) \neq \varnothing$ : We claim that $[0, \ldots, 0] \in \mathcal{M}(\mathbb{T}, g)$, that is there exists an isosceles cusp with particles with boundary $(\mathbb{T}, g)$. Furthermore, faces of this cusp are the faces of the Delaunay tesselation of $(\mathbb{T}, g)$, where the Delaunay tesselation of a surface with a cone metric is defined as the dual of the Voronoi tesselation; see Thurston [32, Proposition 3.1]. To show this, let $T_{D}$ be a Delaunay triangulation, that is a refinement of the Delaunay tesselation of $(\mathbb{T}, g)$. Inscribe a triangle $i j k$ of $T_{D}$ in a horosphere with center $c$. This gives an isosceles semi-ideal pyramid $c i j k$. Let $i j l$ be a triangle of $T_{D}$ adjacent to $i j k$. Develop $i j l$ into the hyperbolic plane spanned by $i j k$. By the main property of Delaunay tesselations, the point $l$ lies outside or on the circumcircle of $i j k$. Hence, $l$ lies outside or on the horosphere through $i, j$ and $k$. It follows that the truncated length of $c l$ is larger than the truncated length of $c i, c j$ and $c k$. In order to make the pyramid $c i j l$ isosceles, one has to rotate the triangle $i j l$ around the edge $i j$ towards $c$. As a result, the total dihedral angle of the pyramids $c i j k$ and $c i j l$ at the edge $i j$ becomes $\leq \pi$.

Property (3): The finiteness of the decomposition (5) is proved in Lemma 3.8. A point $[h] \in \mathbb{R}^{\Sigma} /\langle\mathbf{1}\rangle$ lies on the boundary of $\mathcal{M}^{T}(\mathbb{T}, g)$ if and only if the function $\tilde{h}$ is distance-like across some of the edges of $T$. Clearly, for every edge this condition is analytic. It remains to show that $\mathcal{M}^{T}(\mathbb{T}, g)$ is compact. Embed a triangle $i j k \in \mathcal{T}$ into $\mathbb{H}^{3}$. The hyperbolic plane spanned by $i j k$ divides the sphere at infinity in two 
open hemispheres. The space of semi-ideal pyramids with the base $i j k$ is naturally homeomorphic to any one of these hemispheres. For a point $c \in \partial \overline{\mathbb{H}^{3}}$ not coplanar with $i j k$, call the slope to the pyramid $c i j k$ the maximum distance between the projection of $c$ on the plane spanned by $i j k$ and a point of the triangle $i j k$. Clearly, the slope of $c i j k$ is the maximum slope of the corresponding distance function on $i j k$; see Definition 3.11. For any $D \in \mathbb{R}$, the space of semi-ideal pyramids with slope $\leq D$ is compact. By Lemma 3.12, there exists $D$ such that all pyramids in convex cusps with boundary $(\mathbb{T}, g)$ have slopes $\leq D$. The conditions $\theta_{i j} \leq \pi$ for all $i j \in T$ are closed ones. Thus $\mathcal{M}^{T}(\mathbb{T}, g)$ is compact as a closed subset of a compact space.

Property (2): Let $(T, h)$ be a pair associated with a truncation of $M$. There are two types of edges of $T$ : true edges that are edges of the cusp $M$ and flat edges that were added to refine the face decomposition to a triangulation. We want to show that there exists an $\varepsilon>0$ such that $h^{\prime} \in \mathcal{M}_{\text {tr }}(\mathbb{T}, g)$ for all $h^{\prime}$ in $\mathbb{R}^{\Sigma}$ at a distance $<\varepsilon$ from $h$. That is, for every such $h^{\prime}$ we want to find a triangulation $T^{\prime}$ such that the function $\widetilde{h_{T^{\prime}}^{\prime}}$ exists and is Q-concave.

We obtain a triangulation $T^{\prime}$ from the triangulation $T$ by the flip algorithm. Let $i j$ be an edge of $T$ such that $\widetilde{h_{T}^{\prime}}$ is not Q-concave across $i j$. We call such an edge a bad edge of $T$. If $i j$ belongs to two different triangles $i j k$ and $i j l$ of $T$ and the quadrilateral $i k j l$ is strictly convex, then the edge $i j$ can be flipped, that is replaced by the diagonal $\mathrm{kl}$. The flip algorithm produces a sequence of triangulations $T^{0}=T, T^{1}, T^{2}, \ldots$, where $T^{n+1}$ is obtained from $T^{n}$ by flipping a bad edge of $T^{n}$. If the algorithm terminates at a triangulation $T^{\prime}$ that has no bad edges, then we are done. But some things might go wrong. First, the PD function $\widetilde{h_{T^{n}}^{\prime}}$ might not exist for some $n$. Second, it might be impossible to flip a bad edge; see Figure 4. And third, the algorithm might run infinitely. Let us show that none of these occurs in our particular case.
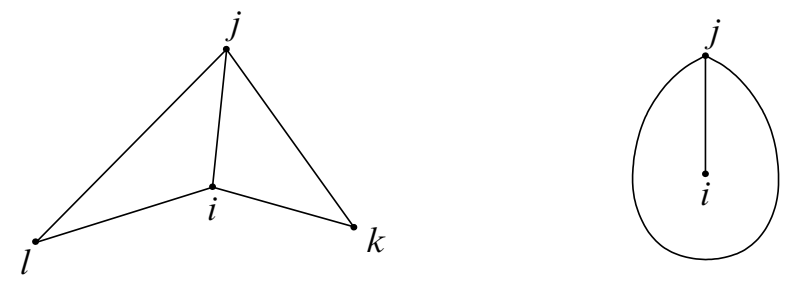

Figure 4: The two situations where the edge $i j$ cannot be flipped

Clearly, the function $\widetilde{h_{T}^{\prime}}$ exists, if $\varepsilon$ is sufficiently small. It is also easy to see that, for small $\varepsilon$, a true edge of $T$ can never become bad and thus will never be flipped. Thus the flip algorithm is performed independently inside every face. This implies that every triangle of $T^{n}$ is contained in a face of $M$, and thus the function $\widetilde{h_{T^{n}}^{\prime}}$ 
exists, for small $\varepsilon$. Since every face is a convex polygon, situations on Figure 4 cannot occur, and a bad edge can always be flipped. The algorithm is finite since the function $\widetilde{h_{T^{n+1}}^{\prime}}$ is pointwise greater or equal than $\widetilde{h_{T^{n}}^{\prime}}$ and since every face has finitely many triangulations.

Property (1): The space $\mathcal{M}(\mathbb{T}, g)$ is compact since it is the union of finitely many compact spaces, by Property (3). The Delaunay cusp constructed at the beginning of the proof has convex faces and thus is an interior point of $\mathcal{M}(\mathbb{T}, g)$ due to Property (2). It remains to prove the convexity of $\mathcal{M}(\mathbb{T}, g)$. We will show that $\mathcal{M}_{\text {tr }}(\mathbb{T}, g)$ is convex, that is for every $h^{0}, h^{1} \in \mathcal{M}_{\mathrm{tr}}(\mathbb{T}, g)$ and every $0<\lambda<1$ the point

$$
h^{\lambda}:=(1-\lambda) h^{0}+\lambda h^{1}
$$

also belongs to $\mathcal{M}_{\mathrm{tr}}(\mathbb{T}, g)$. Then $\mathcal{M}(\mathbb{T}, g)$ is convex as a projection of $\mathcal{M}_{\mathrm{tr}}(\mathbb{T}, g)$.

Since $\mathcal{M}(\mathbb{T}, g)$ is closed, it suffices to prove $h^{\lambda} \in \mathcal{M}_{\mathrm{tr}}(\mathbb{T}, g)$ for all sufficiently small $\lambda$. Our proof of it uses the flip algorithm described in the proof of Property (2).

Let $T$ be a triangulation associated with $h^{0}$. If $\lambda$ is sufficiently small, then the function $\widetilde{h_{T}^{\lambda}}$ exists. The true edges of $T$ will never be flipped, thus the flip algorithm is performed independently inside every face of $\widetilde{h^{0}}$. It follows that for every triangulation $T^{n}$ that appears during the algorithm, the function $\widetilde{h_{T^{n}}^{\lambda}}$ exists. Every face can be triangulated in only finitely many ways, since otherwise infinitely many triangulations would be associated with $h^{0}$, which contradicts Lemma 3.8. Hence the algorithm cannot run infinitely. It remains to show that a bad edge can always be flipped.

Assume that $i j$ is a bad edge in the situation on the left of Figure 4. Badness means

$$
\widetilde{h_{i k}^{\lambda}}(m)<\widetilde{h_{j l}^{\lambda}}(m)
$$

where $m$ is the intersection point of the lines $i k$ and $j l$. On the other hand, by Lemma 3.13 for both $h^{0}$ and $h^{1}$ the opposite inequality (3) holds. By Lemma 3.14, $\widetilde{h_{i k}}(m)$ is a concave and $\widetilde{h_{j l}}(m)$ is a convex function on $h_{i}$ and $h_{k}$. This implies that the inequality (6) is false and $i j$ is not bad. If $i j$ cannot be flipped because of the situation on the right of Figure 4, then the same argument applies since the inequality (4) is a convex condition on $h_{i}$ and $h_{j}$.

Remarks A similar analysis of the space of "generalized" convex polytopes and "generalized" convex caps was made in [6] and [18]. In the former case the distance function was the (square of) the distance from a point to a plane in $\mathbb{R}^{3}$, in the latter case it was the distance from a variable point in a plane to another plane in $\mathbb{R}^{3}$. Thus, instead of $\log \cosh$, the distance-like functions in [6] and [18] were modelled on $x^{2}$ and on $a x$ with a real parameter $a \in[-1,1]$, respectively. In both cases one succeeded to describe 
the space of "generalized" objects explicitly by linear and quadratic inequalities on coordinates.

The arguments from [6] and [18] cannot be carried out in the present paper because the function $\widetilde{h_{i j}}$ from Definition 3.9 does not always exist. As a result, we have less information on $\mathcal{M}(\mathbb{T}, g)$. For example, we have no description of cusps with particles that are boundary points of $\mathcal{M}(\mathbb{T}, g)$.

It is now easy to prove Theorem A in the special case when the metric $g$ has only one singularity. Indeed, the isosceles cusp over the Delaunay tesselation of ( $\mathbb{T}, g)$ is convex; see the first paragraph of the proof of Proposition 3.15. The curvature $\kappa$ of its only particle vanishes due to $\sum_{i} \kappa_{i}=0$. Thus it is a convex polyhedral cusp. This cusp is unique, since the space $\mathcal{M}(\mathbb{T}, g)$ consists of a single point. In the case of one singularity the results of the subsequent sections have no real meaning. If there are two singularities, then the space $\mathcal{M}(\mathbb{T}, g)$ is a segment in $\mathbb{R}$, there can be different triangulations $T$ such that $\mathcal{M}^{T}(\mathbb{T}, g)$ is nonempty, and the things become more interesting.

\section{The discrete total curvature}

In this section we define the discrete total curvature function $S$ on the space $\mathcal{M}(\mathbb{T}, g)$, compute its derivatives and show that $S$ is strictly concave.

\subsection{Derivatives of the discrete total curvature}

Definition 4.1 Let $M \in \mathcal{M}(\mathbb{T}, g)$ be a convex polyhedral cusp with particles. The discrete total curvature of $M$ is defined as

$$
S(M)=-2 \operatorname{Vol}(M)+\sum_{i \in \Sigma} h_{i} \kappa_{i}+\sum_{e \in \mathcal{E}(M)} \ell_{e}\left(\pi-\theta_{e}\right) .
$$

Here $\left(h_{i}\right)_{i \in \Sigma}$ are the truncated particle lengths for an arbitrary truncation of $M, \ell_{e}$ is the length of the edge $e$, and $\theta_{e}$ is the dihedral angle of $M$ at $e$. The second sum ranges over the set $\mathcal{E}(M)$ of edges of $M$.

Choosing a different truncation of $M$ does not change the values of $\kappa_{i}, \ell_{e}$ and $\theta_{e}$. The truncated particle lengths $h_{i}$ are all changed by the same amount. Thus, by Lemma 2.11 , the value $S(M)$ is well-defined. 
Lemma 4.2 The function $S$ is twice continuously differentiable on $\mathcal{M}(\mathbb{T}, g)$. Its first partial derivatives are:

$$
\frac{\partial S}{\partial h_{i}}=\kappa_{i}
$$

The second partial derivatives of $S$ have the properties:

$$
\begin{gathered}
\frac{\partial^{2} S}{\partial h_{i} \partial h_{j}}\left\{\begin{array}{l}
>0 \text { if } i j \in \mathcal{E}(M), \\
=0 \text { otherwise, }
\end{array}\right. \\
\frac{\partial^{2} S}{\partial h_{i}^{2}}=-\sum_{j \neq i} \frac{\partial^{2} S}{\partial h_{i} \partial h_{j}} .
\end{gathered}
$$

Recall that $\mathcal{M}(\mathbb{T}, g) \subset \mathbb{R}^{\Sigma} /\langle\mathbf{1}\rangle$. So, by $\frac{\partial}{\partial h_{i}}$ we mean the directional derivative in the direction of $\left[e_{i}\right]=e_{i}+\langle\mathbf{1}\rangle$, where $e_{i}$ is the $i$-th basis vector of $\mathbb{R}^{\Sigma}$.

Proof Let $T$ be a triangulation of $(\mathbb{T}, g)$ such that $M \in \mathcal{M}^{T}(\mathbb{T}, g)$. Cut $M$ into semi-ideal triangular pyramids according to the triangulation $T$. If $M$ is an interior point of $\mathcal{M}^{T}(\mathbb{T}, g)$, then the triangulation $T$ is associated to all cusps near $M$. By a generalization of the Schläfli formula [22, page 294; 25, Theorem 14.5], we have

$$
d \operatorname{Vol}(M)=\frac{1}{2} \sum_{i} h_{i} d \kappa_{i}-\frac{1}{2} \sum_{e} \ell_{e} d \theta_{e}
$$

This implies (7) in the case when $M$ is an interior point of some $\mathcal{M}^{T}(\mathbb{T}, g)$. In the general case, let $\xi \in \mathbb{R}^{\Sigma}$ be such that $[h+t \xi] \in \mathcal{M}(\mathbb{T}, g)$ for all sufficiently small $t$. Due to the piecewise analyticity of the boundaries of $\mathcal{M}^{T}(\mathbb{T}, g)$, there exists a triangulation $T$ such that $[h+t \xi] \in \mathcal{M}^{T}(\mathbb{T}, g)$ for all sufficiently small $t$. Therefore the previous argument shows that

$$
\frac{\partial S}{\partial \xi}=\sum_{i \in \Sigma} \xi_{i} \kappa_{i}
$$

The same argument can be applied to show that $S \in C^{2}(\mathcal{M}(\mathbb{T}, g))$. Thus we can concentrate on computing the derivatives $\frac{\partial \kappa_{i}}{\partial h_{j}}$ for $M$ in the interior of some $\mathcal{M}^{T}(\mathbb{T}, g)$. This is reduced to computing the derivatives in a single semi-ideal pyramid. Introduce notation for the angles as on Figure 5.

The angle $\omega_{i j k}$ can be viewed as a function of the angles $\rho_{i j}$ and $\rho_{i k}$. Thus we have

$$
\frac{\partial \omega_{i j k}}{\partial h_{j}}=\frac{\partial \omega_{i j k}}{\partial \rho_{i j}} \frac{\partial \rho_{i j}}{\partial h_{j}} .
$$



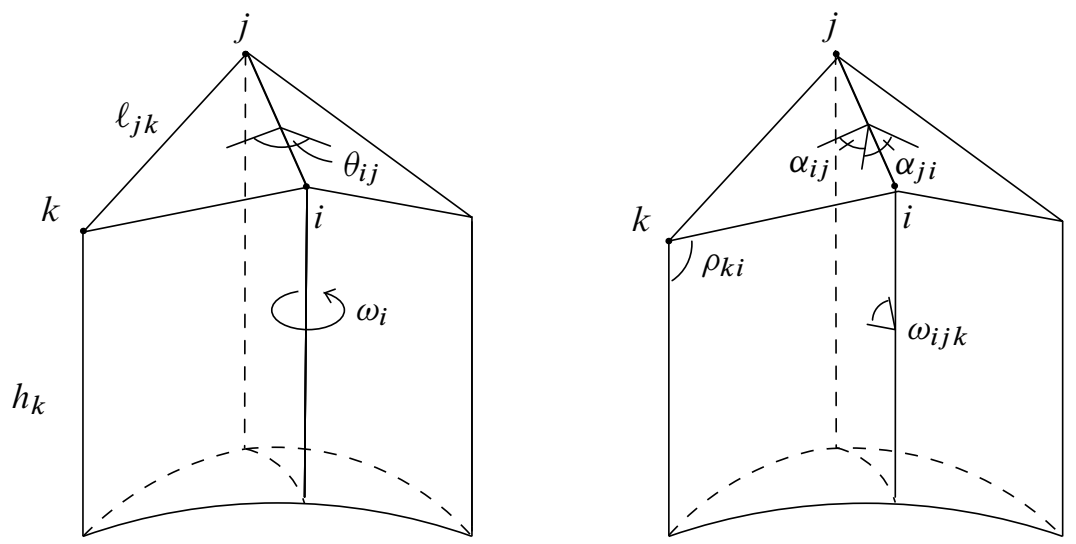

Figure 5: Angles and lengths in two adjacent horoprisms (truncated semiideal pyramids). The curved triangles are the Euclidean bases of the horoprisms.

From (1) we compute

$$
\frac{\partial \rho_{i j}}{\partial h_{j}}=\frac{e^{h_{j}-h_{i}}}{\sinh \ell_{i j} \sin \rho_{i j}} .
$$

From the link of the vertex $i$ in the pyramid over $i j k$ with the help of spherical sine and cosine laws we compute

$$
\frac{\partial \omega_{i j k}}{\partial \rho_{i j}}=-\frac{\cot \alpha_{i j}}{\sin \rho_{i j}} .
$$

Substituting in (10), we obtain

$$
\begin{gathered}
\frac{\partial \omega_{i j k}}{\partial h_{j}}=-\frac{e^{h_{j}-h_{i}} \cot \alpha_{i j}}{\sinh \ell_{i j} \sin ^{2} \rho_{i j}} \\
\frac{\partial \kappa_{i}}{\partial h_{j}}=-\frac{\partial \omega_{i}}{\partial h_{j}}=\frac{e^{h_{j}-h_{i}}\left(\cot \alpha_{i j}+\cot \alpha_{j i}\right)}{\sinh \ell_{i j} \sin ^{2} \rho_{i j}} .
\end{gathered}
$$

Note that $\cot \alpha_{i j}+\cot \alpha_{j i}>0$ if $\alpha_{i j}+\alpha_{j i}<\pi$, and vanishes if $\alpha_{i j}+\alpha_{j i}=\pi$. Since $\alpha_{i j}+\alpha_{j i}=\theta_{i j}$, this implies (8).

The Equation (9) is equivalent to

$$
\sum_{j} \frac{\partial \kappa_{j}}{\partial h_{i}}=0
$$

which is true due to $\sum_{j} \kappa_{j}=0$. 
Remarks Note that the Equation (11) holds only if there is a unique edge between the vertices $i$ and $j$. In the case of multiple edges, one has to sum up the right hand side of (11) over all edges between $i$ and $j$.

In general, $S$ is not of class $C^{3}$. For example, if $\theta_{i j}=\pi$ and $\theta_{i k} \neq \pi$ on Figure 5, then

$$
\frac{\partial}{\partial h_{k}} \frac{\partial \kappa_{i}}{\partial h_{j}}=0 \text {, but } \frac{\partial}{\partial h_{j}} \frac{\partial \kappa_{i}}{\partial h_{k}} \neq 0
$$

\subsection{Concavity of the discrete total curvature}

Let $\Gamma(M)$ be an embedded graph in $(\mathbb{T}, g)$ with vertex set $\Sigma$ and edge set $\mathcal{E}(M)$ that consists of the edges of the convex polyhedral cusp with particles $M$.

Lemma 4.3 The Hessian of the function $S$ is negatively semidefinite. The nullspace of $\left(\frac{\partial^{2} S}{\partial h_{i} \partial h_{j}}\right)$ is spanned by the vectors $v^{K}=\left[h_{i}^{K}\right]_{i \in \Sigma}$ defined as

$$
h_{i}^{K}=\left\{\begin{array}{l}
1, \text { for } i \in K \\
0, \text { for } i \notin K,
\end{array}\right.
$$

where $K \subset \Sigma$ is a connected component of $\Gamma(M)$.

Proof Denote $a_{i j}=\frac{\partial^{2} S}{\partial h_{i} \partial h_{j}}$. Due to (9), we have

$$
\sum_{i, j} a_{i j} x_{i} x_{j}=-\sum_{i<j} a_{i j}\left(x_{i}-x_{j}\right)^{2} .
$$

Since $a_{i j} \geq 0$ by (8), the Hessian is negatively semidefinite. By (8) again, the Hessian vanishes on the vector $x$ if and only if $x_{i}$ is constant over every connected component of $\Gamma(M)$.

As a consequence, $S$ is a concave function on $\mathcal{M}(\mathbb{T}, g)$. Points where the Hessian is degenerate can exist indeed, as the example in Section 2.2 shows. However, in an important special case we can show that the Hessian is nondegenerate.

Lemma 4.4 If $M \in \mathcal{M}(\mathbb{T}, g)$ is a convex polyhedral cusp, then the Hessian of $S$ is nondegenerate at $M$.

Proof The developing map maps $M$ to a convex parabolic polyhedron; see Lemma 2.4. Since the 1-skeleton of a convex polyhedron is connected, the graph $\Gamma(M)$ is also connected. By Lemma 4.3, the nullspace of the Hessian is spanned by the vector $v^{\Sigma}$ which projects to zero in $\mathbb{R}^{\Sigma} /\langle\mathbf{1}\rangle$. 
Note that convex polyhedral cusps correspond to critical points of $S$ due to (7).

Corollary 4.5 If $M \in \mathcal{M}(\mathbb{T}, g)$ is such that $\kappa_{i}(M)=0$ for all $i \in \Sigma$, then

$$
S(M)>S\left(M^{\prime}\right)
$$

for every $M^{\prime} \in \mathcal{M}(\mathbb{T}, g)$ different from $M$.

Although the Hessian of $S$ can degenerate at some points in $\mathcal{M}(\mathbb{T}, g)$, the following lemma holds.

Lemma 4.6 The function $S$ is strictly concave on $\mathcal{M}(\mathbb{T}, g)$, that is

$$
S\left[t h+(1-t) h^{\prime}\right]>t S[h]+(1-t) S\left[h^{\prime}\right]
$$

for all $[h] \neq\left[h^{\prime}\right]$ and all $t \in(0,1)$.

Proof Assume this is not the case. Then there exist $[h] \neq\left[h^{\prime}\right]$ such that $S$ is linear on the segment joining $[h]$ and $\left[h^{\prime}\right]$. By choosing a subsegment, if necessary, we can assume $[h],\left[h^{\prime}\right] \in \mathcal{M}^{T}(\mathbb{T}, g)$ for some triangulation $T$. This implies that the graph $\Gamma=\Gamma[h] \cup \Gamma\left[h^{\prime}\right]$ is embedded in $(\mathbb{T}, g)$.

Due to the linearity of $S$ between $[h]$ and $\left[h^{\prime}\right]$, the vector $\left[h^{\prime}-h\right]$ belongs to the nullspace of the Hessian at both $[h]$ and $\left[h^{\prime}\right]$. Lemma 4.3 implies that the coordinate difference $h_{i}^{\prime}-h_{i}$ is constant over every connected component of the graph $\Gamma$. Thus if $\Gamma$ is connected, then we have $[h]=\left[h^{\prime}\right]$, which is a contradiction.

Let $\Gamma$ be disconnected. Then its complement contains a non-simply connected component $F \subset(\mathbb{T}, g)$. Both functions $\widetilde{h}$ and $\widetilde{h^{\prime}}$ are distance-like functions on $F$ (see Section 3.1). By developing $F$ on a hyperbolic plane in $\mathbb{H}^{3}$, one easily sees that any two distance-like functions of $F$ differ by a constant. This implies that the coordinate difference $h_{i}^{\prime}-h_{i}$ is constant over all vertices of $F$. Since this is true for every nonsimply connected face of $\Gamma$, the difference $h_{i}^{\prime}-h_{i}$ is constant over the whole $\Sigma$, and we have $[h]=\left[h^{\prime}\right]$, which is a contradiction.

\section{Proofs of main theorems}

\subsection{Proof of Theorem A}

Existence Let $M$ be a convex polyhedral cusp with particles and with boundary $(\mathbb{T}, g)$. We will show that if some of its singular curvatures is not zero, then there exists a cusp with a larger discrete total curvature:

$$
\exists i \in \Sigma: \kappa_{i}(M) \neq 0 \quad \Longrightarrow \quad \exists M^{\prime} \in \mathcal{M}(\mathbb{T}, g): S\left(M^{\prime}\right)>S(M) .
$$


Since the space $\mathcal{M}(\mathbb{T}, g)$ is nonempty and compact by Proposition 3.15, the function $S$ attains its maximum at some point $M \in \mathcal{M}(\mathbb{T}, g)$. All of the curvatures of the cusp $M$ must vanish due to (12). Thus (12) implies the existence part of Theorem A.

The proof of (12) is based on:

Lemma 5.1 (Volkov) Let $M$ be a convex polyhedral cusp with particles, and let $\kappa_{i}(M)<0$ for an $i \in \Sigma$. Then every face containing the vertex $i$ has angle $<\pi$ at $i$.

Proof Consider the link of the vertex $i$ in $M$. This is a convex spherical polygon of perimeter $<2 \pi$ with a cone singularity of negative curvature. The lemma says that its boundary cannot contain a geodesic of length $\geq \pi$. This is proved by Volkov in [33]; see also Izmestiev [18, Lemma 9].

Let us prove (12). Since $\sum_{i} \kappa_{i}=0$, we may assume $\kappa_{i}<0$. Due to (7), it suffices to show that the particle length $h_{i}$ can be decreased; in other words, that there exists a cusp $M^{\prime} \in \mathcal{M}(\mathbb{T}, g)$ with truncated particle lengths

$$
\begin{aligned}
& h_{i}^{\prime}=h_{i}-\varepsilon, \\
& h_{j}^{\prime}=h_{j} \quad \text { for every } j \neq i,
\end{aligned}
$$

for a sufficiently small $\varepsilon$. To prove the existence of $M^{\prime}$, we have to find a polyhedral decomposition $\mathcal{F}^{\prime}$ of $(\mathbb{T}, g)$ such that the horoprisms over the faces of $\mathcal{F}^{\prime}$ with heights $h^{\prime}$ exist and their total dihedral angles at the edges of $\mathcal{F}^{\prime}$ are $\leq \pi$.

Consider the face decomposition of $\partial M$. When we decrease $h_{i}$, nothing happens to the horoprisms over the faces that don't contain $i$. Let $F$ be a face of $M$ that contains $i$. By Lemma 5.1, the angle of $F$ at $i$ is $<\pi$. Let $j, k \in \Sigma$ be the vertices of $F$ adjacent to $i$. Draw in $F$ the shortest path $\gamma$ joining $j$ with $k$ and homotopic to the path $j i k$; see Figure 6 . Together $\gamma$ and $j i k$ bound a polygon $P$ that has only three angles $<\pi$, namely those at $i, j$ and $k$. It is not hard to see that subdividing the polygon $P$ by diagonals from $i$ yields a desired decomposition of $(\mathbb{T}, g)$, Figure 6 .

Uniqueness By Corollary 4.5, if $M$ and $M^{\prime}$ are two convex polyhedral cusps with boundary $(\mathbb{T}, g)$, then $S(M)>S\left(M^{\prime}\right)$ and $S\left(M^{\prime}\right)>S(M)$, which is a contradiction.

\subsection{Proof of Theorem C}

Theorem C follows from Lemma 4.6 and the following proposition; see Luo [21, Lemma 6.1] and Izmestiev [18, Proposition 5]. 

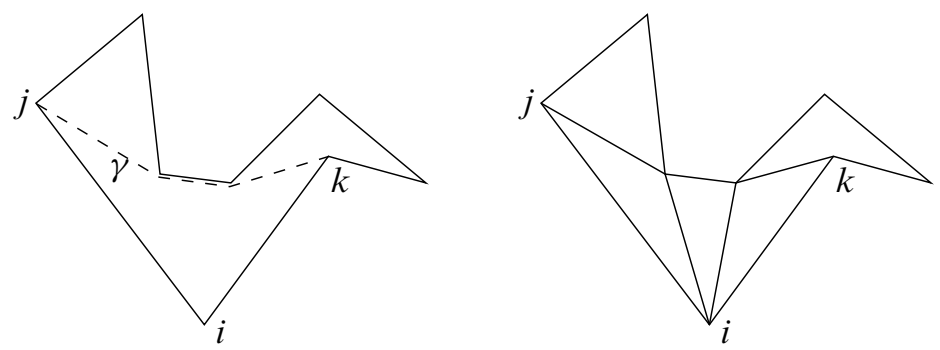

Figure 6: Subdivision of the face $F$ when the height $h_{i}$ decreases

Lemma 5.2 Let $f \in C^{1}(X)$ be a strictly convex or strictly concave function on a compact convex subset $X$ of a vector space $V$. Then the $\operatorname{map} \operatorname{grad} f: X \rightarrow V^{*}$ is a homeomorphism onto the image.

Consider the function $S$ on $\mathcal{M}(\mathbb{T}, g)$. Since $S$ is strictly concave and $\operatorname{grad} S=\kappa$, Theorem $\mathrm{C}$ follows.

Note that cusps with particles are in general not infinitesimally rigid.

\subsection{Proofs of Theorems B, B' and D}

The proofs are based on the fact that the Hessian of $S$ is equal to the Jacobian of the $\operatorname{map}[h] \mapsto \kappa:$

$$
\frac{\partial^{2} S}{\partial h_{i} \partial h_{j}}=\frac{\partial \kappa_{i}}{\partial h_{j}} .
$$

(See (7).) Lemma 4.4 implies that for a cusp with zero curvatures the Jacobian has full rank, that is any nontrivial first-order variation of the particle lengths $[h]$ induces a nontrivial first-order variation of the curvatures $\kappa$.

Recall that a Killing field of hyperbolic space is a vector field of $\mathbb{H}^{3}$ such that the elements of its local 1-parameter group are isometries. An infinitesimal isometric deformation of a polyhedral surface $S$ is a Killing field on each face of a triangulation of $S$ such that two Killing fields on two adjacent triangles are equal on the common edge. The triangulation is required to have the same set of vertices as $S$. An infinitesimal isometric deformation is determined by its values at the vertices of $S$. It is called trivial if it is the restriction to $S$ of a global Killing field. If all the infinitesimal isometric deformations of $S$ are trivial, then $S$ is said to be infinitesimally rigid.

Let $(P, G)$ be a convex parabolic polyhedron. An infinitesimal isometric deformation $Z$ of $\partial P$ is called a parabolic deformation if

$$
Z(g(i))=d g \cdot(Z(i)+\vec{g}(i)),
$$


where $i$ is a vertex of $P$ and $g \in G$. The vector $\vec{g}$ is a parabolic Killing field. It is obtained as follows. Let $g_{t}$ be a path of hyperbolic isometries, leaving invariant the horospheres of same center $c$ as $(P, G)$ and such that $g_{0}=g$. Then

$$
\vec{g}:=\left.d g^{-1} \frac{\partial}{\partial t} g_{t}\right|_{t=0} .
$$

In particular a parabolic Killing field is a Killing field of $\mathbb{H}^{3}$ tangent to all the horospheres of center $c$, and its restriction to each horosphere gives an Euclidean Killing field. A convex parabolic polyhedron is parabolic infinitesimally rigid if all its parabolic deformations are trivial.

Equation (13) arises naturally under the point of view of isometric immersions of surfaces in the hyperbolic space. Due to Lemma 2.4 each convex parabolic polyhedral surface $(\partial P, G)$ is given by a pair $(\varphi, \rho)$, where $\rho$ is a cocompact representation of $\pi_{1}(\mathbb{T})$ in a group of parabolic isometries of $\mathbb{H}^{3}$ and $\varphi$ is a convex polyhedral isometric immersion of the universal cover of $(\mathbb{T}, g)$ in the hyperbolic space, equivariant under the action of $\rho$ : for $x \in \mathbb{R}^{2}$ and $\gamma \in \pi_{1}(\mathbb{T})$,

$$
\varphi(\gamma x)=\rho(\gamma)(\varphi(x)) .
$$

If we derivate a path $\left(\varphi_{t}, \rho_{t}\right)$ of such pairs with respect to $t$, the property of equivariance above leads to Equation (13), where $Z$ arises from the derivative of $\varphi_{t}$, and $\vec{g}$ from the derivative of $\rho_{t}(\gamma)$ (here $g=\rho_{0}(\gamma)$ ). See Fillastre [11] for analogous considerations.

At each vertex $i$ of a convex parabolic polyhedron $(P, G)$ with center $c$ we can decompose $T_{i} \mathbb{H}^{3}$ into a vertical direction that is the direction given by the derivative at $i$ of the ray $c i$, and a horizontal plane, which is orthogonal to the vertical direction. For a vector field $V$ we denote by $V_{v}$ its vertical component and by $V_{h}$ its horizontal component. If $Z$ is a parabolic deformation we have by definition:

$$
Z_{v}(g(i))=d g \cdot Z_{v}(i) .
$$

Roughly speaking, the proof of Theorem B' goes as follows. The radii of a convex parabolic polyhedron are the particle lengths of the corresponding cusp. It follows that each convex parabolic polyhedron is defined by its radii. Hence each parabolic deformation corresponds to a first-order deformation $\dot{r}=\left(\dot{r}_{1}, \ldots, \dot{r}_{n}\right)$ of the radii of the polyhedron such that the corresponding first-order deformation of the singular curvatures $\dot{\kappa}=\left(\dot{\kappa}_{1}, \ldots, \dot{\kappa}_{n}\right)$ is zero: $\dot{r}$ belongs to the nullspace of the Hessian of the discrete total curvature of the cusp. But we know that in this case the nullspace is reduced to a trivial deformation. Such proofs have already been used in cases where no group acts on the deformation $[10 ; 18]$. 
We denote by $\Delta(M)$ the Hessian of the discrete total curvature $S$ at the point $M$.

Lemma 5.3 Let $Z$ be a parabolic deformation of $(\partial P, G)$. Then $Z_{v}$ induces a firstorder deformation $\dot{r}$ of the radii of $(P, G)$ such that $\dot{r}$ belongs to the nullspace of $\Delta(P / G)$.

Proof By definition $Z_{v}$ gives a first-order deformation $\dot{r}$ of the radii of the vertices of $P$. Moreover by Equation (14) this deformation is well-defined on $P / G$. As $Z_{v}$ is an infinitesimal deformation of a polyhedral surface of the hyperbolic space, the angles around the particles of $P / G$ must remain equal to $2 \pi$ under the deformation, that means that $\dot{\kappa}=0$, hence $\dot{r}$ belongs to $\Delta(P / G)$ by (7).

The converse holds:

Lemma 5.4 Let $\dot{r} \in \Delta(P / G)$. Then there exists a unique parabolic deformation $Z$ of $(\partial P, G)$ such that $\dot{r}(i)=\left|Z_{v}(i)\right|$.

Proof In a fundamental domain on $\partial P$ for the action of $G$, for each vertex $i$, we define a vector $Z_{v}(i)$ as the unique vertical vector at $i$ which has norm and direction given by $\dot{r}_{i}$. We define this vector for the other vertices of $P$ using the action of $G$, that defines a vector field $Z_{v}$ on $\partial P$.

The deformation $\dot{r}$ also acts on the projection of the vertices onto a horosphere $H$ of same center $c$ than $(P, G)$. We consider horoprisms given by $H$ together with a triangulation of the faces of $\partial P$. Into each horoprism the deformation $\dot{r}$ gives a horizontal deformation that we call $Z_{h}$. We can extend $Z_{h}$ to $\partial P$ by gluing the horoprisms. The vector field $Z_{h}$ is well-defined because $\dot{r} \in \Delta(P / G)$. Actually we abuse of notation a little, as the vector $Z_{h}$ at a vertex $i$ should be defined as the image under the differential of the orthogonal projection from $H$ to the horosphere concentric to $H$ and passing by $i$ of the vector defined on $H$ that we also denote by $Z_{h}$.

We define the vector field $Z:=Z_{v}+Z_{h}$ on $\partial P$. It is an infinitesimal isometric deformation as it corresponds to a first-order deformations of the particle lengths for a nonvarying boundary metric.

It remains to prove that $Z_{h}$ verifies (13). It will follow that $Z$ verifies (13) because of (14). Consider a fundamental domain on $\partial P$ for the action of $G$. Its projection onto $H$ defines a lattice on $H$ (and hence on $\mathbb{R}^{2}$ ). Applying $Z_{h}$ on this fundamental domain leads to a first-order deformation of the lattice on $H$. Consider a generator $g$ of $G$ given by an edge of the lattice. Let $i$ be a vertex of the lattice (up to project onto $H$ ). Up to compose by a global Killing field suppose that we have $Z_{h}(i)=0$. At 
the vertex $g(i)$, there will be a horizontal first-order displacement, which is given by the deformation of the lattice. Then $Z_{h}(g(i))$ is the restriction to $g(i)$ of a Euclidean Killing field.

Proof of Theorem B' Let $Z$ be a parabolic deformation of a convex parabolic polyhedron $(P, G)$. By the lemmas above $Z$ corresponds to a vector $\dot{r}$ of the nullspace of $\Delta(P / G)$, and by Lemma 4.4 we know that this nullspace is reduced to the trivial deformation 1.

A hyperbolic metric $m$ of a convex polyhedral cusp $M$ can be defined as a section of the bundle of scalar products over $M$. A first-order deformation $\dot{m}$ of $m$ can be defined as a section of the bundle of symmetric bilinear forms over $M$. Such a deformation is trivial if it is given by the Lie derivative of $g$ under the action of a vector field of $M$. We will only consider deformations $\dot{m}$ such that the metric remains hyperbolic, ie the first-order variation of the sectional curvature of $m$ induced by $\dot{m}$ vanishes. The deformation $\dot{m}$ is trivial on $\partial M$ if its restriction to $\partial M$ is zero.

Definition 5.5 A convex polyhedral cusp $M$ is called infinitesimally rigid if every deformation $\dot{m}$ of $M$ which is trivial on $\partial M$ is trivial on the whole $M$.

Proof of Theorem B It is known that a first-order deformation of a hyperbolic manifold with convex boundary which is trivial on the boundary is equivalent to an equivariant infinitesimal isometric deformation of the image of the boundary by the developing map. Moreover one is trivial when the other is; see eg Schlenker [30]. It follows that Theorem B is equivalent to Theorem B'.

Proof of Theorem D We sketch the proof as it is word by word the same as in the Section 4 of [27], even if this reference concerns Euclidean polytopes. The idea of the proof is the same as for convex parabolic polyhedra above: it is sufficient to prove that the matrix $\Delta$ associated to each weakly convex star-shaped parabolic polyhedra is negatively definite (the definition of the discrete total curvature doesn't use the convexity). And this follows directly from the case of convex parabolic polyhedra as:

(1) a weakly convex star-shaped parabolic polyhedron $P$ is obtained from a convex parabolic polyhedra $P^{\prime}$ by removing a finite number of simplices (in a fundamental domain) [27, Lemma 4.1];

(2) each time we remove a simplex, the matrix $\Delta^{\prime}$ associated to $P^{\prime}$ changes by the addition of a negatively semidefinite matrix [27, Lemmas 4.3 and 4.4];

(3) at the end $\Delta$ is negatively definite as $\Delta^{\prime}$ is [27, Lemma 4.5]. 


\section{References}

[1] A Alexandroff, Existence of a convex polyhedron and of a convex surface with a given metric, Rec. Math. [Mat. Sbornik] N.S. 11(53) (1942) 15-65 MR0013540

[2] A D Alexandrov, Convex polyhedra, Springer Monographs in Math., Springer, Berlin (2005) MR2127379 Translated from the 1950 Russian edition by N S Dairbekov, S S Kutateladze and A B Sossinsky, With comments and bibliography by V A Zalgaller and appendices by L A Shor and Yu A Volkov

[3] M T Anderson, Scalar curvature and the existence of geometric structures on 3manifolds. I, J. Reine Angew. Math. 553 (2002) 125-182 MR1944810

[4] E M Andreev, Convex polyhedra in Lobačevskĭ spaces, Mat. Sb. (N.S.) 81 (123) (1970) 445-478 MR0259734

[5] W Blaschke, G Herglotz, Über die Verwirklichung einer geschlossenen Fläche mit vorgeschriebenem Bogenelement im Euklidischen Raum, Sitzungsber. Bayer. Akad. Wiss., Math.-Naturwiss. Abt. 2 (1937) 229-230

[6] A I Bobenko, I Izmestiev, Alexandrov's theorem, weighted Delaunay triangulations, and mixed volumes, Ann. Inst. Fourier (Grenoble) 58 (2008) 447-505 MR2410380

[7] F Bonsante, J-M Schlenker, AdS manifolds with particles and earthquakes on singular surfaces, To appear in Geom. Funct. Anal. arXiv:math/0609116

[8] R D Canary, D B A Epstein, P L Green, Notes on notes of Thurston [MR0903850], from: "Fundamentals of hyperbolic geometry: selected expositions", London Math. Soc. Lecture Note Ser. 328, Cambridge Univ. Press (2006) 1-115 MR2235710 With a new foreword by Canary

[9] A-L Cauchy, Sur les polygones et polyèdres (Second Mémoire), from: “Evres complètes, Seconde série 1" (1905) 26-38

[10] R Connelly, J-M Schlenker, On the infinitesimal rigidity of weakly convex polyhedra arXiv:math/0606681

[11] F Fillastre, Fuchsian polyhedra in Lorentzian space-forms arXiv: math.DG/0702532

[12] F Fillastre, Polyhedral realisation of hyperbolic metrics with conical singularities on compact surfaces, Ann. Inst. Fourier (Grenoble) 57 (2007) 163-195 MR2313089

[13] F Fillastre, Polyhedral hyperbolic metrics on surfaces, Geom. Dedicata 134 (2008) 177-196 MR2399657

[14] F Fillastre, I Izmestiev, Dual metrics of hyperbolic cusps with convex polyhedral boundary, In preparation (2008)

[15] CD Hodgson, Deduction of Andreev's theorem from Rivin's characterization of convex hyperbolic polyhedra, from: "Topology '90 (Columbus, OH, 1990)", Ohio State Univ. Math. Res. Inst. Publ. 1, de Gruyter, Berlin (1992) 185-193 MR1184410 
[16] C D Hodgson, I Rivin, A characterization of compact convex polyhedra in hyperbolic 3-space, Invent. Math. 111 (1993) 77-111 MR1193599

[17] C Indermitte, T M Liebling, M Troyanov, H Clémençon, Voronoi diagrams on piecewise flat surfaces and an application to biological growth, from: "Combinatorics and computer science (Palaiseau, 1997)”, Theoret. Comput. Sci. 263 (2001) 263-274 MR1846934

[18] I Izmestiev, A variational proof of Alexandrov's convex cap theorem, To appear in Discrete Comput. Geom. arXiv:math/0703169

[19] K Krasnov, J-M Schlenker, Minimal surfaces and particles in 3-manifolds, Geom. Dedicata 126 (2007) 187-254 MR2328927

[20] F Labourie, J-M Schlenker, Surfaces convexes fuchsiennes dans les espaces lorentziens à courbure constante, Math. Ann. 316 (2000) 465-483 MR1752780

[21] F Luo, Rigidity of polyhedral surfaces arXiv:math.GT/0612714

[22] J Milnor, Collected papers. Vol. 1. Geometry, Publish or Perish, Houston, TX (1994) MR1277810

[23] S Moroianu, J-M Schlenker, Quasi-Fuchsian manifolds with particles arXiv: math/0603441

[24] I Rivin, On geometry of convex polyhedra in hyperbolic 3-space, $\mathrm{PhD}$ thesis, Princeton University (1986)

[25] I Rivin, Euclidean structures on simplicial surfaces and hyperbolic volume, Ann. of Math. (2) 139 (1994) 553-580 MR1283870

[26] J-M Schlenker, Hyperbolic manifolds with polyhedral boundary Available at http:// www.picard.ups-tlse.fr/ schlenker/texts/ideal.pdf

[27] J-M Schlenker, On weakly convex star-shaped polyhedra arXiv:0704.2901

[28] J-M Schlenker, Surfaces convexes dans des espaces lorentziens à courbure constante, Comm. Anal. Geom. 4 (1996) 285-331 MR1393565

[29] J-M Schlenker, Convex polyhedra in Lorentzian space-forms, Asian J. Math. 5 (2001) 327-363 MR1868937

[30] J-M Schlenker, Hyperbolic manifolds with convex boundary, Invent. Math. 163 (2006) 109-169 MR2208419

[31] S Sechelmann, Alexandrov polyhedron editor Available at http:// www.math.tu-berlin.de/geometrie/ps/software.shtml

[32] W P Thurston, Shapes of polyhedra and triangulations of the sphere, from: "The Epstein birthday schrift”, (I Rivin, C Rourke, C Series, editors), Geom. Topol. Monogr. 1 (1998) 511-549 MR1668340

[33] Y A Volkov, Existence of a polyhedron with a given development, $\mathrm{PhD}$ thesis, Leningrad State University (1955) In Russian 
[34] Y A Volkov, Existence of convex polyhedra with prescribed development I, Vestn. Leningr. Univ. 15 (1960) 75-86

Département de Mathématiques, Université de Cergy-Pontoise / Saint-Martin 2, avenue Adolphe Chauvin, 95302 Cergy-Pontoise Cedex, France Institut für Mathematik, MA 8-3, Technische Universität Berlin Str. des 17. Juni 136, D-10623 Berlin, Germany francois.fillastre@u-cergy.fr, izmestiev@math.tu-berlin.de

Proposed: Jean-Pierre Otal

Seconded: Walter Neumann, Benson Farb
Received: 14 December 2007 Revised: 7 October 2008 